\title{
Public Health Expenditures and Health Outcomes in Pakistan: Evidence from Quantile Autoregressive Distributed Lag Model
}

\author{
Irfan Ullah' \\ Assad Ullah (D) ${ }^{2}$ \\ Sher Ali $\mathbb{D}^{3}$ \\ Petra Poulova $\mathbb{D D}^{4}$ \\ Ahsan Akbar (iD ${ }^{5}$ \\ Muhammad Haroon Shah ${ }^{6}$ \\ Alam Rehman ${ }^{7}$ \\ Muhammad Zeeshan ${ }^{8}$ \\ Fakhr E Alam Afridi (iD ${ }^{3}$ \\ 'Reading Academy, Nanjing University of \\ Information Science and Technology, \\ Nanjing, People's Republic of China;

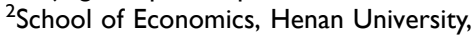 \\ Kaifeng, People's Republic of China; \\ ${ }^{3}$ Department of Economics, Islamia \\ College Peshawar, Peshawar, Pakistan; \\ ${ }^{4}$ Department of Informatics and \\ Quantitative Methods, Faculty of \\ Informatics and Management, University \\ of Hradec Kralove, Hradec Kralove, 500 \\ 03, Czech Republic; ${ }^{5}$ International \\ Business School, Guangzhou City \\ University of Technology, Guangzhou, \\ 510080, People's Republic of China; \\ ${ }^{6}$ College of International Students, Wuxi \\ University, Wuxi 214105, Jiangsu, \\ People's Republic of China; ${ }^{7}$ Faculty of \\ Management Sciences, National \\ University of Modern Languages, \\ Islamabad, Pakistan; ${ }^{8}$ College of Business \\ Administration, Liaoning Technical \\ University, XingCheng, Liaoning Province, \\ 125 105, People's Republic of China
}

Correspondence: Ahsan Akbar International Business School, Guangzhou City University of Technology,

Guangzhou, 510080, People's Republic of China

Email akbar@gcu.edu.cn
Objective: The provision of healthcare facilities remains high on the manifesto of various political parties in Pakistan and healthcare spending has witnessed a significant surge in the last two decades that is expected to positively influence health outcomes in the country. Therefore, this research aims to explore the effects of healthcare expenditures on the actual health status of the masses in Pakistan for the period 1995Q1 to 2017Q1.

Methods: We apply the Quantile Autoregressive Distributed Lag (QARDL) approach for estimation purposes. This is the most recent and emerging estimation technique in time series analysis.

Results: Our findings confirm that public healthcare spending significantly impacts health outcomes in Pakistan both in the short-run and long-run. Public healthcare spending improves life expectancy and reduces death rate and infant mortality.

Conclusion: The study concludes that public healthcare is the main focus of the current regime. It is noticed that spending on healthcare significantly contributes to the health outcomes in Pakistan. These efforts by the government significantly promote life expectancy and drop down the mortality ratio in the country. Based on these notable facts, the government should allocate sufficient resources towards the latest healthcare technologies and equipment to optimize health outcomes in the country.

Keywords: healthcare spending, health outcomes, healthcare technologies, Quantile cointegration, QARDL, Pakistan

\section{Introduction}

Healthcare spending is considered an integral part of the economy and significantly contributes to the economic development. The conventional endogenous growth models suggest that human capital growth may positively affect the output per worker in the long run. ${ }^{1}$ Grossman's human capital model presents that health quality significantly influences human capital development via additional working time and utility derived from good health. ${ }^{2}$ Healthy labor leads to higher earnings in the short run but improves life expectancy as well in the long run. ${ }^{3}$ Mentally and physically healthy persons can channelize their energy into effective production. According to Somi, Butler, Vahid, Njau, Abdulla, ${ }^{4}$ good health conditions have both short-run and long-run outcomes, ie, in the short-run good health leads to higher productivity, which ultimately yields higher earnings in the long-run. Both industrialization and global competition have resulted in environmental degradation, thereby leading to poor health conditions. Besides, industrial production 
heavily relies on fossil fuels, which are the biggest contributor to environmental pollution, leading to adversely affected human health. ${ }^{5}$ The study conducted by Ostro and Rothschild ${ }^{6}$ revealed that small particulate matters may result in work loss and even lead to bed disability in adults. Schwartz and Dockery ${ }^{7}$ reported that contaminants, such as total suspended particulate (TSP) and sulfur dioxide, increase human death chances. The effect of environmental damage in $\mathrm{CO} 2$ emissions on health has substantial consequences for healthcare expenditures.

The majority of the previous research reported that different factors responsible for healthcare spending include health status, insurance coverage, income, and demographic characteristics. ${ }^{8}$ Among the above, the income factor is considered as the main determinant of health expenditures. Additionally, $\mathrm{CO}_{2}$ and a poor environment are also core factors affecting health spending. $\mathrm{CO}_{2}$ is considered an externality that can have potentially negative consequences for labor productivity. It has direct implications for industrial performance and economic growth. Apergis et $\mathrm{al}^{9}$ and Hansen, Selte ${ }^{10}$ confirmed that small particulate matter (SPM) raises the probability of sick leaves and adversely affects the respiratory system of humans. Jerrett et $\mathrm{al}^{11}$ examined whether countries with higher pollution had been exposed to more health hazards, which resulted in higher health spending. Chaabouni and Saidi ${ }^{12}$ found a causal relationship between $\mathrm{CO}_{2}$ emissions and healthcare spending and concluded that greater $\mathrm{CO}_{2}$ emissions lead to higher healthcare spending. Many other studies such as Brunekreef, Holgate; ${ }^{13}$ Mead, Brajer; ${ }^{14}$ Narayan, Narayan; ${ }^{15}$ Janke, Propper, Henderson; ${ }^{16}$ Remoundou, Koundouri ${ }^{17}$ and Beatty, Shimshack ${ }^{18}$ have confirmed a positive association between air pollution and healthcare spending.

Healthcare spending is generally considered to improve health outcomes such as life expectancy, death rates, and mortality. There are mixed findings on health spending and health outcomes; Akinkugbe and Mohanoe ${ }^{19}$ analyzed the health care expenditures are positively related to health outcomes in Lesotho. Novignon et al's ${ }^{20}$ results show that health care expenditure significantly influences health status through improving life expectancy, reducing death and infant mortality rates in sub-Saharan African countries. Both public and private health care spending showed a strong positive association with health status furthermore, public health care spending had a relatively higher impact. Similarly, Ventelou and Abu-Zaineh ${ }^{21}$ argued that health spending positively affects health outcomes in the
MENA region. Arthur and Oaikhenan ${ }^{22}$ found that health expenditure significantly affects health outcomes in subSaharan Africa. Ssozi, Amlani ${ }^{23}$ and Behera, Dash ${ }^{24}$ reported that health spending improves Southeast Asian countries' health outcomes.

Conversely, some studies 'did not prove a significant association between healthcare expenditures and outcomes. For example, Dollar and Burnside ${ }^{25}$ found an insignificant relationship between health expenditures and infant mortality in low-income countries. Filmer ${ }^{2}$ also reported an insignificant impact on healthcare spending on health outcomes. Healthcare expenditures are essential for developing countries, and most of the population has a lack of healthcare facilities. Besides developing countries, the population may face serious health risks due to the poor healthcare system. The most common diseases experienced in developing countries are Cardio-vascular diseases, Cancer, Diabetes, Diarrheal diseases, HIV/AIDS, Malaria, Stroke and Tuberculosis, etc., which leads to an increase in deaths in developing countries. According to Bokhari et $\mathrm{al}^{26}$ approximately 11 million children particularly infants die annually in developing countries.

Although health spending has an increasing trend in Pakistan's economic history, health outcomes are not properly explored in the extant literature. Therefore, the present study aims to investigate the health spending and health outcomes in Pakistan from 1995Q1 to 2017Q4. This research contributes to the existing body of knowledge from the following aspects; first, most of the previous studies employ a linear methodology for the analysis, which does not capture the dynamic behavior of the variables; therefore, we apply Quantile Autoregressive Distributed Lag Model (QARDL) to uncover the dynamic nexus between healthcare spending and the resulting health outcomes in Pakistan.

Secondly, we used both short and long estimations to observe the aforementioned association, which enables us to understand the short-term and long-term implications of health spending on health outcomes. Lastly, we used the time period from 1995Q1-2017Q4 that cover the health spending by both military and democratic regimes. This enables us to unveil the health care priorities and achievements during different political regimes.

Against the backdrop of sluggish economic performance, the government has a limited cushion to escalate health care spending in Pakistan. The policy implications of this research entail that health care spending significantly promotes life expectancy and reduce the mortality 
rate in the country. Hence, the policymakers shall explore new avenues of investment in the health sector. Encouraging public-private partnerships and providing a conducive environment for private investors can lower the health spending burden of the government as well as boost the quality of life of the masses in the country.

\section{Health Expenditures in Pakistan}

Healthcare spending improves human capital. ${ }^{27}$ However, developing countries often pay less attention to health expenditures in the annual national budget (WHO, 2010). Akram and $\mathrm{Khan}^{28}$ reported healthcare facilities and health spending are unequally distributed, particularly people in rural areas face the lack of healthcare facilities. In contrast, less healthcare spending is allocated for the rural areas. Furthermore, Akram and $\mathrm{Khan}^{28}$ reported that health spending positively affects economic growth in the long run, but in the short run, it has a negative association. Chaudhry et $\mathrm{al}^{29}$ found that health improvement in health statuses such as life expectancy and mortality significantly contributed to the economic growth in Pakistan, and the government may achieve economic development goals by increasing health spending.

Table 1 represents the trends of health care expenditure in Pakistan from 1995 to 2015. In 1995, the total health expenditures were $2.05 \%$, which is at the lowest level, it gradually increases to 3.02 in 2010 , which remained the same till 2005. Private healthcare expenditures were lowest

Table I Health Expenditure (HE) Trends in Pakistan

\begin{tabular}{|c|c|c|c|c|}
\hline Years & $\begin{array}{c}\text { HE Total } \\
\text { (\% of } \\
\text { GDP) }\end{array}$ & $\begin{array}{c}\text { HE Private } \\
\text { (\% of GDP) }\end{array}$ & $\begin{array}{c}\text { HE Public } \\
\text { (\% of GDP) }\end{array}$ & $\begin{array}{c}\text { HE per Capita } \\
\text { (Current US\$) }\end{array}$ \\
\hline 1995 & 2.05 & 1.85 & 26.18 & 15.77 \\
2000 & 2.78 & 2.18 & 21.83 & 15.49 \\
2005 & 2.91 & 2.23 & 23.54 & 22.36 \\
2010 & 3.01 & 2.06 & 31.64 & 30.95 \\
2015 & 3.01 & 1.82 & 36.40 & 36.40 \\
\hline
\end{tabular}

Abbreviation: WDI, World Development Index. in 1995 which increased in 2005, and gradually declined thereafter and reached up to the lowest share of $1.8 \%$ in 2015. Similarly, public health expenditures were at the lowest, $2.18 \%$ in 2000 , and a significant increase of $3.64 \%$ in 2015. Table 2 reports population and health indicators in Pakistan from 1995 to 2015. Children with age 0-14 were highest in portion, and it contributed to $42.7 \%$ of the total population, which declined and reached to lowest level 35\% of the total population in 2015. While young with age 15-60 accounted for $53.3 \%$ of the total population in 1995 , which increased and reached $60.5 \%$ in 2015 . It implies that $60 \%$ of the population of Pakistan are young, which are assumed to have good health. The death rate was 9.6 per thousand, which was the highest ratio in 1995 and declined gradually in subsequent years, and it reached 7.3 per thousand till the end of 2015. Similarly, the mortality rate had remained high in 1995 , which was 97.1 per thousand and it declined gradually to 65.8 per thousand in 2015 .

\section{Materials and Methods}

\section{The Model}

$$
\begin{gathered}
L E_{t}=\beta_{o}+\beta_{1} \ln G D P_{t}+\beta_{2} H E_{t}+\beta_{3} \text { Pop }_{t}+\varepsilon_{1 t} \\
D R_{t}=\beta_{o}+\beta_{1} \ln G D P_{t}+\beta_{2} H E_{t}+\beta_{3} \text { Pop }_{t}+\varepsilon_{2 t} \\
M_{t}=\beta_{o}+\beta_{1} \ln G D P_{t}+\beta_{2} H E_{t}+\beta_{3} \text { Pop }_{t}+\varepsilon_{3 t}
\end{gathered}
$$

Where,

LE is the life expectancy

$\mathrm{DR}$ is the death rate

$\mathrm{M}$ is used for the molarity

lnGDP shows the log of GDP

HE is the health expenditures

Pop is population

$\varepsilon_{2 t}, \varepsilon_{2 t}, \varepsilon_{3 t}$ shows the error for the corresponding equations.

Model 1-3 presents the health outcomes, such as LE, $\mathrm{DR}$, and $\mathrm{M}$ with health spending HE, while GDP and

Table 2 Population Health Indicator in Pakistan

\begin{tabular}{|l|c|c|c|c|c|c|}
\hline Years & $\begin{array}{c}\text { Ages 0-14 } \\
\text { (\% of Total) }\end{array}$ & $\begin{array}{c}\text { Ages 15-64 } \\
\text { (\% of Total) }\end{array}$ & $\begin{array}{c}\text { Ages Above 65 } \\
\text { (\% of Total) }\end{array}$ & $\begin{array}{c}\text { Life Expectancy } \\
\text { Total (Years) }\end{array}$ & $\begin{array}{c}\text { Death Rate } \\
\text { (People/ 1 000) }\end{array}$ & $\begin{array}{c}\text { Mortality Rate } \\
\text { Live/ I000 }\end{array}$ \\
\hline 1995 & 42.71 & 53.30 & 3.99 & 61.44 & 9.68 & 97.10 \\
2000 & 41.10 & 54.81 & 4.10 & 62.73 & 8.73 & 87.70 \\
2005 & 38.18 & 57.56 & 4.26 & 63.84 & 7.16 & 80.00 \\
2010 & 36.18 & 59.40 & 4.43 & 65.13 & 7.37 & 73.50 \\
2015 & 35.01 & 60.50 & 4.49 & 66.32 & 6.80 \\
\hline
\end{tabular}

Abbreviation: WDI, World Development Index. 
population are taken as explanatory variables. Equation 3 shows three main outcomes discussed in the literature: life expectancy, death rate, and infant mortality. The increase in public health spending is expected to promote life expectancy due to availability of better health facilities for the general public. However, the association of population with life expectancy is still unclear in the extant literature. The second equation illustrates that e-death rate and an increase in health spending reduce the death rate, which implies a negative expected sign. The logic behind this is attributed to the fact that availability of better health infrastructure provides the necessary support to fight against deadly diseases. Equation 3 shows the association between public health spending and infant mortality. An increase in health expenditures will reduce infant mortality due to better health and support infrastructure. Likewise, GDP is expected to have a positive linkage with life expectancy and a negative link with mortality and death rate, while the population has a positive expected relationship with the death rate. The GDP and Population are used as control variables. The selected control variables in our models are based on existing literature such as Ssozi, Amlani; ${ }^{23}$ Behera, Dash $;{ }^{24}$ Novignon et $a l ;{ }^{20}$ Ventelou, Abu-Zaineh ${ }^{21}$ and Arthur, Oaikhenan. ${ }^{22}$ These studies examined the health outcomes and health spending relationship by adding both population and GDP as control variables in their models. The results of this study are in line with those of Osakede, ${ }^{30}$ Arthur, Oaikhenan, ${ }^{22}$ Ssozi, Amlani, ${ }^{23}$ and Nicholas et al. ${ }^{31}$

\section{Methodology}

We need a suitable statistical or econometric tool to compute the impact of independent variables on the dependent variable; we used the Quantile-Autoregressive-Distributive -Lag method for the empirical analysis. This approach explains the possible asymmetry (differences) in the response of one variable to changes in another variable over a range of different quantiles. The QARDL methodology is preferred because conventional OLS and linear ARDL techniques are based on conditional mean, and it does not provide detailed information for different quantiles. Healthcare expenditures may affect differently in different quantiles on the health status; therefore, QARDL provides results that varied across the different quantiles. Pakistan's economy has been through political regimes and preferences on health spending have been varied and deviating in different regimes. It may have a diverse impact on health outcomes. Therefore, to capture the dynamic behavior of public health spending in the different periods, we use the Quantile ARDL method to capture it, which could provide more stable results in different quantiles. QARDL provides stable outcomes as compared to the linear models due to the following reasons.

(i) It provides a nonlinear relationship between health spending and health outcomes variables in the short and long run.

(ii) The QARDL estimates whether healthcare expenditure affects health outcomes in each of the conditional quantiles.

Quantile Auto Regressive Distributed Lag (QARDL) is an appropriate method to examine the quantile-dependent relationship comprising a mixed order of integration of included variables in the model. ${ }^{32}$ The QARDL is an extension of the Pesaran and $\mathrm{Shin}^{33}$ mechanism, and the dependent variables' extreme tail of distribution can be estimated in the QARDL framework. We follow the initial ARDL process, which is a first-generation model.

Auto-Regressive Distributed Lag is defined as

$$
Y_{t}=\alpha_{*}+\sum_{j=1}^{p} \phi_{j^{*}} Y_{t-j}+\sum_{j=0}^{q} \theta_{j *} X_{t-j}+U_{t}
$$

Where $X_{t} \in \Re^{k}$, is the stationary and ergodic process of integration by assuming the zero population mean. $U_{t}$, is the error term of the model, which is further elaborated as $Y_{t}-E\left[Y \mid F_{t-1}\right]$ with $F_{t-1}$ the smallest $\sigma$ - field process, which is obtained by $\left\{X_{t}, Y_{t-1}, X_{t-1}, \ldots\right\}$, with $\mathrm{p}$ and $\mathrm{q}$ are lag orders, respectively. We also consider the assumption that $k$ variables in $X_{t}$ are not cointegrated with each other. Based on this assumption, the $\tau$ quantile of $Y_{t}$ conditional on $\quad F_{t-1} \quad$ is described as $\quad \alpha_{*}(\tau)+\sum_{j=1}^{p} \phi_{j *}(\tau) Y_{t-j}+$ $\sum_{j=0}^{p} \theta_{j *}(\tau) X_{t-j}$ and are shown to be $Q_{Y_{t}}\left(\tau \mid F_{t-1}\right) . Y_{t}$ can be explained by the following equation;

$$
Y_{t}=\alpha_{*}(\tau)+\sum_{j=1}^{p} \phi_{j *}(\tau) Y_{t-j}+\sum_{j=0}^{p} \theta_{j *}(\tau) X_{t-j}+U_{t}(\tau)
$$

Equation 5 is the standard form of quantile autoregressive distributed lag (QARDL) model, and $U_{t}(\tau)$ above is $Y_{t}-Q_{Y_{t}}\left(\tau \mid F_{t-1}\right)$.

To analyze the process of QARDL, eq. (5) can be written as follows 


$$
Y_{t}=\alpha_{*}(\tau)+\sum_{j=0}^{q-1} W_{t-j} \delta_{j *}(\tau) X_{t} \gamma_{*}+\sum_{j=0}^{p} \theta_{j *}(\tau) Y_{t-j}+U_{t}(\tau)
$$

Where $\gamma_{*}(\tau):=\sum_{j=0}^{p} \theta_{j *}(\tau) X, W_{t}:=\Delta X_{t}$, and $\delta_{j *}(\tau):=-\sum_{i=j+1}^{q} \theta_{i *}(\tau)$. For the value given $\tau \in(0,1)$, the short-run dynamics measuring parameter can be estimated by minimization problem, which is given as: $\min \sum_{t} \rho_{t}\left(Y_{t}-\alpha-\sum_{j=o}^{q-1} W_{t} \delta_{j}(\tau)+\sum_{j=1}^{p} \phi_{j} Y_{t-j}\right) \quad$ where $\rho_{t}(u)=u(\tau-I(u<0))$ is the check function.

The estimate of the long-run parameters by taking the plug-in principle is explained in Eq. (7) as follows:

$$
\hat{\beta}(\tau)=\hat{\gamma}(\tau)\left(1-\sum_{j=1}^{p} \phi_{j}(\tau)\right)^{-1}
$$

In order to obtain more clear-sightedness by referring to Zho et al, we use the following $E\left(\rho_{t}\left(U_{t}(\tau)\right)\right)=0$ and the QARDL is written as:

$$
\begin{aligned}
Q_{\Delta Y_{t}}(\tau \mid .)= & \alpha(\tau)+\zeta(\tau)\left(Y_{t-1}-X_{t-1} \beta(\tau)\right)+\sum_{j=1}^{p-1} \tilde{\phi}_{j}(\tau) Y_{i-j} \\
& +\sum_{j=0}^{q-1} \Delta X_{t-j} \lambda_{j}(\tau)
\end{aligned}
$$

Where

$$
\zeta(\tau)=\sum_{j=1}^{p} \phi_{j}(\tau)-1, \lambda_{0}(\tau)=\gamma(\tau)+\delta_{0}(\tau), \tilde{\phi}_{j}(\tau)
$$

$$
=-\sum_{i=j+1}^{p} \phi_{i}(\tau)
$$

and $\lambda_{j}(\tau)=-\sum_{i=j+1}^{q} \delta_{j}(\tau)$

for $j=1,2, \ldots$

Chao et al explain some interesting aspects of these equations; It shows that short- and long-run parameters depend on quantile. This signifies that the QARLD parameter can be different through quantiles, meaning that it is affected by quantiles the innovation $U_{t}(\tau)$ received at each period. Zero correlation between regressors and the error is the main condition for having a constant quantiledependent cointegration vector by Xiao. It allows for quantile-dependent cointegrating parameters only under the dynamic OLS approach, using leads and lags, but not under the semi-parametric fully modified framework. ${ }^{34}$ The data for all the variables are obtained from the World Bank database online from 1995 to 2017, converted into quarter frequency following Sbia et al. Shahbaz et al ${ }^{35}$ by using the quadratic match-sum method.

\section{Results}

\section{ADF Unit Root Test}

ADF unit root test is the first test applied in the cointegration procedure. However, there is no strict order of integration for QARDL. Table 3 contains the ADF unit root test; the first column shows variable names. The second and third columns present estimations both at the level and at the difference, while the last column shows the order of integration. Life expectancy is stationary at the level where death rate and mortality are found to be stationary at the first difference. In contrast, GDP, health expenditure, and HIV are found to be non-stationary at the level and stationary at the second difference. The ADF unit root test shows that the variables of our model have a mixed order of integration.

\section{Quantile ARDL Outcome}

The QARDL outcomes of some specific quantiles for the life expectancy, death rate, and morality are reported in Tables 4-6 respectively. In all the tables, the error correction is reported by $\rho$ parameter, while $\beta$ is used for shortrun parameters, and long-run coefficients are reported by

Table 3 Unit Root Test Results

\begin{tabular}{|l|c|c|c|}
\hline Variable & At Level & At First Difference & Order of Stationarity (Decision) \\
\hline Life Expectancy & $-3.539542^{* *}(2.573784)$ & $\mathrm{NA}$ & $\mathrm{I}(0)$ \\
Death Rate & $3.507570^{* *}(-2.573784)$ & $-6.464770^{* *}(-1.942035)$ & $\mathrm{I}(\mathrm{I})$ \\
Mortality & $-2.103034^{* * *}(-1.942035)$ & & $\mathrm{I}(\mathrm{I})$ \\
Gross Domestic Product & $0.716322^{* * *}(-1.942035)$ & $-57.44254^{*}(-1.942035)$ & $\mathrm{I}(2)$ \\
Health Expenditure & $0.501232^{* * *}(-1.942035)$ & $-61.20754 * *(-1.942035)$ & $\mathrm{I}(2)$ \\
HIV & $\mathrm{I} .005646(-85.79524)$ & $-1.942035^{* *}(-1.942035)$ & $\mathrm{I}(2)$ \\
\hline
\end{tabular}

Notes: The values in without parenthesis are the augmented dickey Fuller test-statistic values and the values in parentheses in columns 2 nd and $3 r d$ are Test-statistic critical values. Values in parentheses in column 4 rth are order of stationarity $* 10 \%$ level of significance. $* * 5 \%$ significance. *** $1 \%$ level of significance. The values in parenthesis are representing standard errors. 
Table 4 Dependent Variable Life Expectancy

\begin{tabular}{|c|c|c|c|c|c|c|c|c|c|}
\hline Quantile $(\tau)$ & $\rho(\tau)$ & $\beta_{*}^{G D P}(\tau)$ & $\beta_{*}^{H E}(\tau)$ & $\beta_{*}^{H I V}(\tau)$ & $\beta_{*}^{P o p}(\tau)$ & $\omega_{*}^{G D P}(\tau)$ & $\omega_{*}^{H E}(\tau)$ & $\omega_{*}^{H I V}(\tau)$ & $\omega_{*}^{P o p}(\tau)$ \\
\hline 0.100 & $\begin{array}{c}0.429923 \\
(0.195474)\end{array}$ & $\begin{array}{c}0.027 \mid 28 \\
(0.001427)\end{array}$ & $\begin{array}{c}0.002999 \\
(0.00017 I)\end{array}$ & $\begin{array}{r}-0.000608 \\
(5.80 \mathrm{E}-05)\end{array}$ & $\begin{array}{l}-0.047268 \\
(0.001233)\end{array}$ & $\begin{array}{l}-2.129088 \\
(1.436259)\end{array}$ & $\begin{array}{c}0.200897 \\
(0.132313)\end{array}$ & $\begin{array}{c}0.00334 I \\
(0.01426 I)\end{array}$ & $\begin{array}{c}0.739387 \\
(0.485750)\end{array}$ \\
\hline 0.200 & $\begin{array}{c}0.599442 \\
(0.283208)\end{array}$ & $\begin{array}{c}0.025214 \\
(0.001852)\end{array}$ & $\begin{array}{c}0.00295 I \\
(0.000235)\end{array}$ & $\begin{array}{l}-0.000531 \\
(7.34 \mathrm{E}-05)\end{array}$ & $\begin{array}{l}-0.049562 \\
(0.001663)\end{array}$ & $\begin{array}{l}-1.811548 \\
(1.153534)\end{array}$ & $\begin{array}{c}0.161586 \\
(0.101908)\end{array}$ & $\begin{array}{c}0.005783 \\
(0.011962)\end{array}$ & $\begin{array}{c}0.6834 I I \\
(0.416949)\end{array}$ \\
\hline 0.300 & $\begin{array}{c}0.532650 \\
(0.277857)\end{array}$ & $\begin{array}{c}0.026754 \\
(0.001926)\end{array}$ & $\begin{array}{c}0.002883 \\
(0.000226)\end{array}$ & $\begin{array}{l}-0.000598 \\
(7.19 E-05)\end{array}$ & $\begin{array}{l}-0.049238 \\
(0.001652)\end{array}$ & $\begin{array}{l}-3.686584 \\
(2.947062)\end{array}$ & $\begin{array}{c}0.328953 \\
(0.082556)\end{array}$ & $\begin{array}{l}-0.012912 \\
(0.009976)\end{array}$ & $\begin{array}{c}\text { I.329683 } \\
(0.35374 \text { I) }\end{array}$ \\
\hline 0.400 & $\begin{array}{c}0.819 \mid 23 \\
(0.688337)\end{array}$ & $\begin{array}{c}0.018759 \\
(0.012307)\end{array}$ & $\begin{array}{c}0.001756 \\
(0.001657)\end{array}$ & $\begin{array}{l}-0.000330 \\
(0.000379)\end{array}$ & $\begin{array}{l}-0.055|3| \\
(0.003745)\end{array}$ & $\begin{array}{l}-3.158809 \\
(2.790954)\end{array}$ & $\begin{array}{c}0.273942 \\
(0.064915)\end{array}$ & $\begin{array}{l}-0.009318 \\
(0.008727)\end{array}$ & $\begin{array}{c}1.214295 \\
(0.318762)\end{array}$ \\
\hline 0.500 & $\begin{array}{c}1.040919 \\
(0.479939)\end{array}$ & $\begin{array}{c}0.004582 \\
(0.002223)\end{array}$ & $\begin{array}{c}0.000762 \\
(0.000249)\end{array}$ & $\begin{array}{c}\text { I.82E-05 } \\
(5.20 \mathrm{E}-05)\end{array}$ & $\begin{array}{l}-0.051180 \\
(0.002403)\end{array}$ & $\begin{array}{l}-4.591612 \\
(3.459564)\end{array}$ & $\begin{array}{c}0.405255 \\
(0.038545)\end{array}$ & $\begin{array}{l}-0.024809 \\
(0.005261)\end{array}$ & $\begin{array}{c}1.725777 \\
(0.191427)\end{array}$ \\
\hline 0.600 & $\begin{array}{c}0.425866 \\
(0.042004)\end{array}$ & $\begin{array}{c}0.003024 \\
(0.001926)\end{array}$ & $\begin{array}{c}0.000858 \\
(0.000296)\end{array}$ & $\begin{array}{c}3.22 \mathrm{E}-05 \\
(4.94 \mathrm{E}-05)\end{array}$ & $\begin{array}{l}-0.050162 \\
(0.002662)\end{array}$ & $\begin{array}{l}-4.855636 \\
(3.289609)\end{array}$ & $\begin{array}{c}0.402331 \\
(0.021608)\end{array}$ & $\begin{array}{l}-0.03066 \mathrm{I} \\
(0.003892)\end{array}$ & $\begin{array}{c}2.007401 \\
(0.151780)\end{array}$ \\
\hline 0.700 & $\begin{array}{c}0.095021 \\
(0.277923)\end{array}$ & $\begin{array}{c}0.000935 \\
(0.001180)\end{array}$ & $\begin{array}{c}0.001704 \\
(0.000360)\end{array}$ & $\begin{array}{c}3.5 \mathrm{IE}-05 \\
(4.28 \mathrm{E}-05)\end{array}$ & $\begin{array}{l}-0.052658 \\
(0.003 / 85)\end{array}$ & $\begin{array}{l}-5.048504 \\
(4.201986)\end{array}$ & $\begin{array}{c}0.442556 \\
(0.013708)\end{array}$ & $\begin{array}{l}-0.033567 \\
(0.002927)\end{array}$ & $\begin{array}{c}2.010893 \\
(0.145764)\end{array}$ \\
\hline 0.800 & $\begin{array}{l}-0.109353 \\
(0.2464 \mid 4)\end{array}$ & $\begin{array}{c}0.000178 \\
(0.001034)\end{array}$ & $\begin{array}{c}0.002201 \\
(0.000376)\end{array}$ & $\begin{array}{c}4.14 \mathrm{E}-05 \\
(4.17 \mathrm{E}-05)\end{array}$ & $\begin{array}{l}-0.056540 \\
(0.003217)\end{array}$ & $\begin{array}{l}-4.82 \mid 485 \\
(3.206735)\end{array}$ & $\begin{array}{c}0.421623 \\
(0.012092)\end{array}$ & $\begin{array}{l}-0.031275 \\
(0.002924)\end{array}$ & $\begin{array}{c}1.929508 \\
(0.150216)\end{array}$ \\
\hline 0.900 & $\begin{array}{c}0.008792 \\
(0.019109)\end{array}$ & $\begin{array}{c}0.000305 \\
(0.000806)\end{array}$ & $\begin{array}{c}0.002502 \\
(0.000340)\end{array}$ & $\begin{array}{c}5.90 \mathrm{E}-05 \\
(3.40 \mathrm{E}-05)\end{array}$ & $\begin{array}{l}-0.059683 \\
(0.002949)\end{array}$ & $\begin{array}{l}-5.103085 \\
(3.172355)\end{array}$ & $\begin{array}{c}0.450506 \\
(0.011506)\end{array}$ & $\begin{array}{l}-0.036136 \\
(0.002743)\end{array}$ & $\begin{array}{c}2.070263 \\
(0.162762)\end{array}$ \\
\hline
\end{tabular}

Table 5 Dependent Variable Death Rate

\begin{tabular}{|c|c|c|c|c|c|c|c|c|c|}
\hline Quantile $(\tau)$ & $\rho(\tau)$ & $\beta_{*}^{G D P}(\tau)$ & $\beta_{*}^{H E}(\tau)$ & $\beta_{*}^{H I V}(\tau)$ & $\beta_{*}^{P o p}(\tau)$ & $\omega_{*}^{G D P}(\tau)$ & $\omega_{*}^{H E}(\tau)$ & $\omega_{*}^{H I V}(\tau)$ & $\omega_{*}^{P o p}(\tau)$ \\
\hline 0.100 & $\begin{array}{l}-0.063647 \\
(0.018784)\end{array}$ & $\begin{array}{l}-0.000538 \\
(0.001097)\end{array}$ & $\begin{array}{l}-0.002101 \\
(0.000425)\end{array}$ & $\begin{array}{c}0.000186 \\
(4.26 \mathrm{E}-05)\end{array}$ & $\begin{array}{c}0.113845 \\
(0.203657)\end{array}$ & $\begin{array}{l}-4.101354 \\
(0.137379)\end{array}$ & $\begin{array}{l}-0.362023 \\
(0.009563)\end{array}$ & $\begin{array}{c}0.028864 \\
(0.002229)\end{array}$ & $\begin{array}{l}-1.600100 \\
(0.133299)\end{array}$ \\
\hline 0.200 & $\begin{array}{l}-0.152419 \\
(0.221404)\end{array}$ & $\begin{array}{l}-0.000426 \\
(0.001293)\end{array}$ & $\begin{array}{l}-0.001740 \\
(0.000446)\end{array}$ & $\begin{array}{c}0.000175 \\
(4.87 E-05)\end{array}$ & $\begin{array}{c}0.110874 \\
(0.303805)\end{array}$ & $\begin{array}{l}-3.886860 \\
(0.181829)\end{array}$ & $\begin{array}{l}-0.341286 \\
(0.009860)\end{array}$ & $\begin{array}{c}0.024465 \\
(0.002668)\end{array}$ & $\begin{array}{l}-1.465560 \\
(0.138979)\end{array}$ \\
\hline 0.300 & $\begin{array}{c}0.126408 \\
(0.250699)\end{array}$ & $\begin{array}{l}-0.002417 \\
(0.001921)\end{array}$ & $\begin{array}{l}-0.001037 \\
(0.000393)\end{array}$ & $\begin{array}{c}0.000 I 49 \\
(5.7 I E-05)\end{array}$ & $\begin{array}{c}0.107297 \\
(0.703419)\end{array}$ & $\begin{array}{l}-4.059040 \\
(0.174896)\end{array}$ & $\begin{array}{l}-0.356084 \\
(0.011098)\end{array}$ & $\begin{array}{c}0.026406 \\
(0.002536)\end{array}$ & $\begin{array}{l}-1.536818 \\
(0.127790)\end{array}$ \\
\hline 0.400 & $\begin{array}{c}0.359523 \\
(0.065563)\end{array}$ & $\begin{array}{l}-0.004920 \\
(0.003678)\end{array}$ & $\begin{array}{c}5.97 \mathrm{E}-05 \\
(0.000528)\end{array}$ & $\begin{array}{c}0.000165 \\
(5.00 \mathrm{E}-05)\end{array}$ & $\begin{array}{c}0.107177 \\
(0.804856)\end{array}$ & $\begin{array}{l}-3.661576 \\
(0.316079)\end{array}$ & $\begin{array}{l}-0.307834 \\
(0.023348)\end{array}$ & $\begin{array}{c}0.021525 \\
(0.004338)\end{array}$ & $\begin{array}{l}-1.425380 \\
(0.168932)\end{array}$ \\
\hline 0.500 & $\begin{array}{c}1.071545 \\
(0.446 \mid 96)\end{array}$ & $\begin{array}{l}-0.008164 \\
(0.003282)\end{array}$ & $\begin{array}{c}0.000207 \\
(0.000265)\end{array}$ & $\begin{array}{c}0.000123 \\
(6.66 \mathrm{E}-05)\end{array}$ & $\begin{array}{c}0.110171 \\
(0.202823)\end{array}$ & $\begin{array}{r}-3.711775 \\
(0.389879)\end{array}$ & $\begin{array}{l}-0.327016 \\
(0.032938)\end{array}$ & $\begin{array}{c}0.019878 \\
(0.004526)\end{array}$ & $\begin{array}{l}-|.32888| \\
(0.164308)\end{array}$ \\
\hline 0.600 & $\begin{array}{c}0.99046 \mathrm{I} \\
(0.747974)\end{array}$ & $\begin{array}{l}-0.034286 \\
(0.020623)\end{array}$ & $\begin{array}{l}-0.002131 \\
(0.002735)\end{array}$ & $\begin{array}{c}0.000525 \\
(0.000619)\end{array}$ & $\begin{array}{c}0.120105 \\
(0.605886)\end{array}$ & $\begin{array}{l}-2.532108 \\
(0.661776)\end{array}$ & $\begin{array}{l}-0.219350 \\
(0.054031)\end{array}$ & $\begin{array}{c}0.007265 \\
(0.007373)\end{array}$ & $\begin{array}{r}-0.911454 \\
(0.270354)\end{array}$ \\
\hline 0.700 & $\begin{array}{c}0.519948 \\
(0.307797)\end{array}$ & $\begin{array}{l}-0.045929 \\
(0.002947)\end{array}$ & $\begin{array}{l}-0.004005 \\
(0.000393)\end{array}$ & $\begin{array}{c}0.000925 \\
(0.000110)\end{array}$ & $\begin{array}{c}0.112636 \\
(0.672827)\end{array}$ & $\begin{array}{l}-2.484033 \\
(0.756520)\end{array}$ & $\begin{array}{l}-0.227102 \\
(0.064899)\end{array}$ & $\begin{array}{c}0.005254 \\
(0.007732)\end{array}$ & $\begin{array}{l}-0.809675 \\
(0.278161)\end{array}$ \\
\hline 0.800 & $\begin{array}{c}0.541943 \\
(0.326891)\end{array}$ & $\begin{array}{l}-0.043504 \\
(0.002937)\end{array}$ & $\begin{array}{l}-0.004129 \\
(0.000448)\end{array}$ & $\begin{array}{c}0.000832 \\
(0.000117)\end{array}$ & $\begin{array}{c}0.113095 \\
(0.113154)\end{array}$ & $\begin{array}{r}-1.065623 \\
(0.607697)\end{array}$ & $\begin{array}{l}-0.095799 \\
(0.053456)\end{array}$ & $\begin{array}{c}0.009320 \\
(0.006388)\end{array}$ & $\begin{array}{l}-0.325476 \\
(0.221980)\end{array}$ \\
\hline 0.900 & $\begin{array}{c}0.486391 \\
(0.248800)\end{array}$ & $\begin{array}{l}-0.045475 \\
(0.002254)\end{array}$ & $\begin{array}{l}-0.004172 \\
(0.000337)\end{array}$ & $\begin{array}{c}0.000912 \\
(9.28 \mathrm{E}-05)\end{array}$ & $\begin{array}{l}0.111046 \\
(0.28448)\end{array}$ & $\begin{array}{r}-1.748934 \\
(I .15749 I)\end{array}$ & $\begin{array}{l}-0.162452 \\
(0.106923)\end{array}$ & $\begin{array}{c}0.002984 \\
(0.011542)\end{array}$ & $\begin{array}{l}-0.536348 \\
(0.391954)\end{array}$ \\
\hline
\end{tabular}


Table 6 Dependent Variable Infant Mortality Rate

\begin{tabular}{|c|c|c|c|c|c|c|c|c|c|}
\hline Quantile $(\tau)$ & $\rho(\tau)$ & $\beta_{*}^{G D P}(\tau)$ & $\beta_{*}^{H E}(\tau)$ & $\beta_{*}^{H I V}(\tau)$ & $\beta_{*}^{P o p}(\tau)$ & $\omega_{*}^{G D P}(\tau)$ & $\omega_{*}^{H E}(\tau)$ & $\omega_{*}^{H I V}(\tau)$ & $\omega_{*}^{P o p}(\tau)$ \\
\hline 0.100 & $\begin{array}{r}-0.083094 \\
(0.088438)\end{array}$ & $\begin{array}{l}-0.002045 \\
(0.001690)\end{array}$ & $\begin{array}{l}-0.006102 \\
(0.000657)\end{array}$ & $\begin{array}{c}0.000546 \\
(6.25 E-05)\end{array}$ & $\begin{array}{l}-0.029968 \\
(0.003993)\end{array}$ & $\begin{array}{l}-35.9704 \mid \\
(I .2 \mid 6772)\end{array}$ & $\begin{array}{r}-3.176780 \\
(0.07985 I)\end{array}$ & $\begin{array}{c}0.255199 \\
(0.019244)\end{array}$ & $\begin{array}{r}-14.88357 \\
(1.138470)\end{array}$ \\
\hline 0.200 & $\begin{array}{l}-0.096765 \\
(0.012515)\end{array}$ & $\begin{array}{c}-0.008121- \\
(0.005772)\end{array}$ & $\begin{array}{l}-0.004357 \\
(0.001229)\end{array}$ & $\begin{array}{c}0.000491 \\
(9.62 E-05)\end{array}$ & $\begin{array}{l}-0.031185 \\
(0.003695)\end{array}$ & $\begin{array}{l}-34.02356 \\
(1.47 \mid 538)\end{array}$ & $\begin{array}{r}-2.977186 \\
(0.085399)\end{array}$ & $\begin{array}{c}0.221175 \\
(0.020822)\end{array}$ & $\begin{array}{r}-13.89887 \\
(1.070566)\end{array}$ \\
\hline 0.300 & $\begin{array}{c}0.145298 \\
(0.253177)\end{array}$ & $\begin{array}{l}-0.014279 \\
(0.005793)\end{array}$ & $\begin{array}{l}-0.002685 \\
(0.000844)\end{array}$ & $\begin{array}{c}0.000505 \\
(0.000109)\end{array}$ & $\begin{array}{l}-0.026729 \\
(0.005223)\end{array}$ & $\begin{array}{l}-35.53320 \\
(1.439955)\end{array}$ & $\begin{array}{r}-3.115350 \\
(0.095445)\end{array}$ & $\begin{array}{c}0.236612 \\
(0.020869)\end{array}$ & $\begin{array}{l}-14.44923 \\
(1.043670)\end{array}$ \\
\hline 0.400 & $\begin{array}{c}0.116727 \\
(0.281072)\end{array}$ & $\begin{array}{l}-0.018248 \\
(0.006549)\end{array}$ & $\begin{array}{l}-0.001063 \\
(0.000734)\end{array}$ & $\begin{array}{c}0.000438 \\
(7.32 \mathrm{E}-05)\end{array}$ & $\begin{array}{l}-0.010148 \\
(0.011461)\end{array}$ & $\begin{array}{c}34.09208 \\
(2.092594)\end{array}$ & $\begin{array}{l}-2.826645 \\
(0.156191)\end{array}$ & $\begin{array}{c}0.215278 \\
(0.028 \mid 50)\end{array}$ & $\begin{array}{l}-14.38745 \\
(1.097621)\end{array}$ \\
\hline 0.500 & $\begin{array}{c}0.153305 \\
(0.077108)\end{array}$ & $\begin{array}{l}-0.03215 \mathrm{I} \\
(0.029079)\end{array}$ & $\begin{array}{l}-0.001524 \\
(0.001174)\end{array}$ & $\begin{array}{c}0.000694 \\
(0.000475)\end{array}$ & $\begin{array}{c}0.008976 \\
(0.016700)\end{array}$ & $\begin{array}{l}-32.49027 \\
(3.202239)\end{array}$ & $\begin{array}{r}-2.863699 \\
(0.268680)\end{array}$ & $\begin{array}{c}0.177708 \\
(0.0367 / 8)\end{array}$ & $\begin{array}{l}-12.55072 \\
(1.336 \mid 43)\end{array}$ \\
\hline 0.600 & $\begin{array}{c}1.099326 \\
(0.835003)\end{array}$ & $\begin{array}{l}-0.160554 \\
(0.059551)\end{array}$ & $\begin{array}{l}-0.013966 \\
(0.007456)\end{array}$ & $\begin{array}{c}0.003872 \\
(0.001705)\end{array}$ & $\begin{array}{c}0.070101 \\
(0.019709)\end{array}$ & $\begin{array}{l}-22.45008 \\
(5.501130)\end{array}$ & $\begin{array}{l}-1.94398 I \\
(0.45 \mid 42 I)\end{array}$ & $\begin{array}{c}0.069400 \\
(0.060827)\end{array}$ & $\begin{array}{l}-8.975328 \\
(2.221833)\end{array}$ \\
\hline 0.700 & $\begin{array}{c}0.675083 \\
(0.024038)\end{array}$ & $\begin{array}{l}-0.201294 \\
(0.011867)\end{array}$ & $\begin{array}{l}-0.021604 \\
(0.001887)\end{array}$ & $\begin{array}{c}0.005206 \\
(0.000416)\end{array}$ & $\begin{array}{c}0.055480 \\
(0.010473)\end{array}$ & $\begin{array}{l}-25.59508 \\
(6.294619)\end{array}$ & $\begin{array}{r}-2.282755 \\
(0.549649)\end{array}$ & $\begin{array}{c}0.08777 \mid \\
(0.0663 \mid 7)\end{array}$ & $\begin{array}{l}-9.534297 \\
(2.346790)\end{array}$ \\
\hline 0.800 & $\begin{array}{c}0.743162 \\
(0.262555)\end{array}$ & $\begin{array}{l}-0.193853 \\
(0.012618)\end{array}$ & $\begin{array}{l}-0.021900 \\
(0.002399)\end{array}$ & $\begin{array}{c}0.004875 \\
(0.000467)\end{array}$ & $\begin{array}{c}0.060013 \\
(0.014560)\end{array}$ & $\begin{array}{l}-|1.6837| \\
(8.07|| 60)\end{array}$ & $\begin{array}{l}-1.045261 \\
(0.713808)\end{array}$ & $\begin{array}{r}-0.050633 \\
(0.083715)\end{array}$ & $\begin{array}{l}-4.732090 \\
(2.914158)\end{array}$ \\
\hline 0.900 & $\begin{array}{c}0.878724 \\
(0.263891)\end{array}$ & $\begin{array}{l}-0.193755 \\
(0.011932)\end{array}$ & $\begin{array}{l}-0.021595 \\
(0.002676)\end{array}$ & $\begin{array}{c}0.004893 \\
(0.00047 I)\end{array}$ & $\begin{array}{c}0.065877 \\
(0.018179)\end{array}$ & $\begin{array}{l}-15.35969 \\
(10.01286)\end{array}$ & $\begin{array}{r}-1.438325 \\
(0.923033)\end{array}$ & $\begin{array}{l}-0.019002 \\
(0.099525)\end{array}$ & $\begin{array}{r}-5.677708 \\
(3.3874 \mid 4)\end{array}$ \\
\hline
\end{tabular}

$\omega$. The error correction parameter shows mixed results: most of the coefficients hold a positive sign, except the 8th quantile, which appears with a negative sign. Besides the majority of the coefficient's signs are significant except 4th, 7th and 9th quantile, which is insignificant. These results imply the existence of a long-run relationship. Furthermore, the positive sign of the coefficient depicts the divergence property, which means that the short-run parameters of the model will take a longer time to achieve the long-run equilibrium.

Table 4 presents the first model results of QARDL in which we use life expectancy as a dependent variable. The short-run and long-run estimations show a mix result; GDP is associated positively and significantly with life expectancy. GDP in 2nd 3rd and 5th quantile significantly influences life expectancy, while 4th, 6th, 7th has an insignificant association. Similarly, in the long run, estimations of only the 1 st and 2 nd quantiles show a significant effect on life expectancy, while the remaining quantile 3rd to 9th quantile shows an insignificant effect. The health expenditures show a positive and significant association in all quantiles except the 4th quantile, which reports an insignificant coefficient.
Similarly, in the long run, 1st and 2nd quantiles do not significantly affect life expectancy. However, the $3 \mathrm{rd}$ to 9th quantile shows that health expenditures coefficients are significant at 5\% level. In the short run, HIV shows a mixed result; in the 1 st to 3 rd quantile, the HIV coefficient is negatively related to life expectancy, while HIV coefficients reported an insignificant effect on life expectancy, while in the long-run from 6th quantile to 9th quantile HIV and a significant negative effect on life expectancy. The population is negatively related to life expectancy in the short-run for all quantiles. While the long-run estimations show mixed results, some coefficients are positive, while some are negatively associated with life expectancy.

Table 5 contains results for the model in which the death rate is the dependent variable, while explanatory variables are the same as the first model. Most short-run coefficients show a negative association between death rate and GDP but 1st to 4th quantiles do not have a significant coefficient. Similarly, the long-run coefficients are also negatively related to the death rate. Health expenditures are also negatively associated with the death rate both in the short run and long run. HIV has a positive effect on the death rate both in 
the short run and long run. The population shows a mixed result both in the short and long runs; furthermore, most of the coefficients are insignificant both in the short and long runs. Table 6 shows quantile ARDL results for a model in which mortality is used as a dependent variable. GDP per capita is negatively related to mortality, from 5th to 9th quantile show significant coefficients of GDP. In the long run, 3rd to 7th quantiles have a significant and negative association between mortality and GDP. Likewise, health spending also reported a negative and significant association with mortality in most of the quantiles. In contrast, the longrun results did not significantly influence health spending on mortality. HIV shows a positive effect on mortality both in the short-run and long-run. The population has negatively influenced the molarity both in the short and long runs, and all the coefficients are significant at 5\% level.

\section{Rolling Window QARDL Results}

The rolling window framework estimates the QARDL model time variations in the quantile-specific impact of life expectancy, death rate, and mortality. Figures 1-3 plots the series of the rolling quantile estimates of the parameters GDP, HE, HIV Pop for life expectancy, death rate, and mortality, respectively, at different confidence levels. The quantile estimates display a strong timevarying pattern signifying that independent variables have a different impact on the dependent variable. We also estimated the heterogeneity of the quantiles by using the Wald test. Figures 4-6 represent rolling hypothesis estimates of time-varying p-values of the Wald test. First, we observe a significant time dependence in all parameter estimates. Secondly, the P-values of the Wald test show a significant impact of the included variables in Figures 4-6. We address the heterogeneity of the health status variables to their determinants, including healthcare spending. These findings suggest that healthcare spending asymmetries exist across the selected quantiles.

\section{Discussion}

\section{ADF Unit Root}

In cointegration, the first step is to know the stationary properties of the variables, and the ADF unit root test provides stationary properties of the variables. The Augmented Dickey-Fuller ${ }^{36}$ unit root test is applied to determine the order of integration. Although quantile ARDL regression does not restrict to the order of integration, QARDL can be applied if variables have mixed order of integration. Table 3 reports the ADF unit root test of the included variables. Life expectancy, death rate, and mortality are non-stationary at level and become stationary at the first difference. GDP per capita, health expenditures, and HIV are non-stationary at the first level. However, they became stationary at the second difference. These outcomes confirm that our variables have mixed integration order. Hence, the conventional cointegration test suggested by $^{37}$ is conditioned to have a unique integration order for all the variables included in the model.

\section{Quantile ARDL Results}

The short-run and long-run estimations reported mixed results; GDP in the short-run has a positive and significant effect on the life expectancy in 1st, 2nd, 3rd, and 5th quantile while 4 th to 7 th quantile has an insignificant influence on GDP per capita. This indicates the GDP has little impact on life expectancy. However, the long-run effect is heterogeneous, and the initial 1st and 2nd quantiles show a significant effect on life expectancy. While the rest of the quantiles show a negative and insignificant impact. Healthcare spending positively affects life expectancy in all quantiles except the 4th quantile where it is insignificant. This implies that public healthcare spending can significantly improve life expectancy, for example, in the 3 rd quantile a one percent increase in public healthcare spending increases the life expectancy by $0.002 \%$. The long-run estimations reported that healthcare spending has no influence on the first two quantiles, while the remaining quantiles show a significant effect of healthcare spending on the life expectancy, for example, in the 4th quantile, the reported long-run estimations show that each percent increase in healthcare spending increases the life expectancy by the $0.27 \%$. This implies that healthcare spending is the main determinant of life expectancy both in the short and long-run and the higher health spending provides better health facilities to the people and thus people may live longer and have a longer life expectancy.

The HIV results significantly negative influence on life expectancy in the first three quantiles. However, from the 4th to the 9th quantile, HIV is insignificant. In the long run, the HIV has the last five quantiles and has a significantly negative effect on life expectancy; for example, in the 7th quantile, the increase in HIV cases reduces the life expectancy by $0.03 \%$. This implies little influence of HIV on the determination of life expectancy. The relationship between HIV and life expectancy is insignificant in most quantiles, this is mainly due to low rates of 

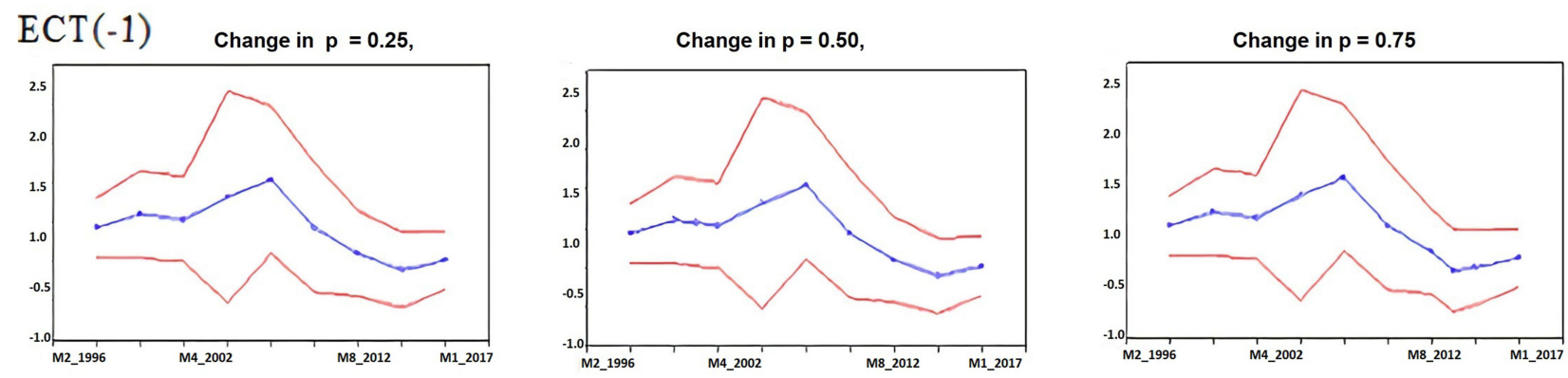

\section{GDP}
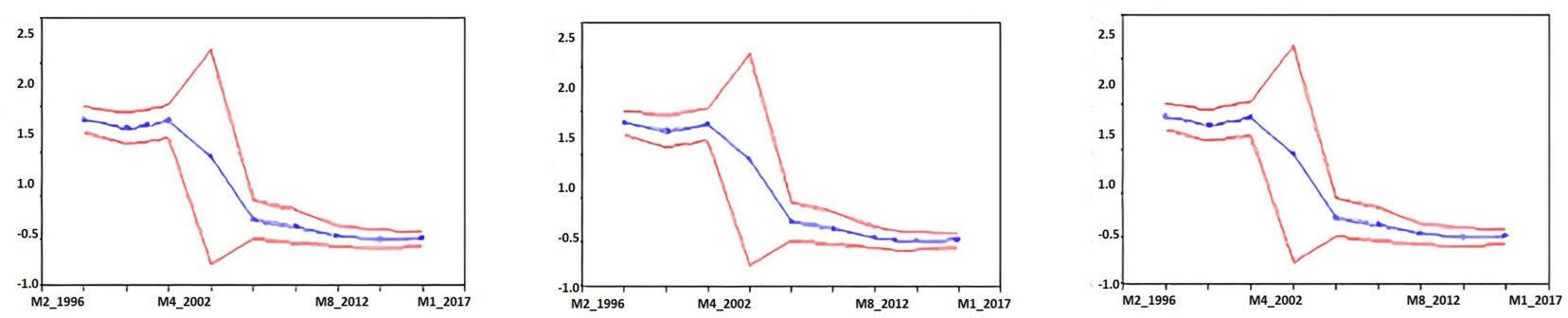

HE
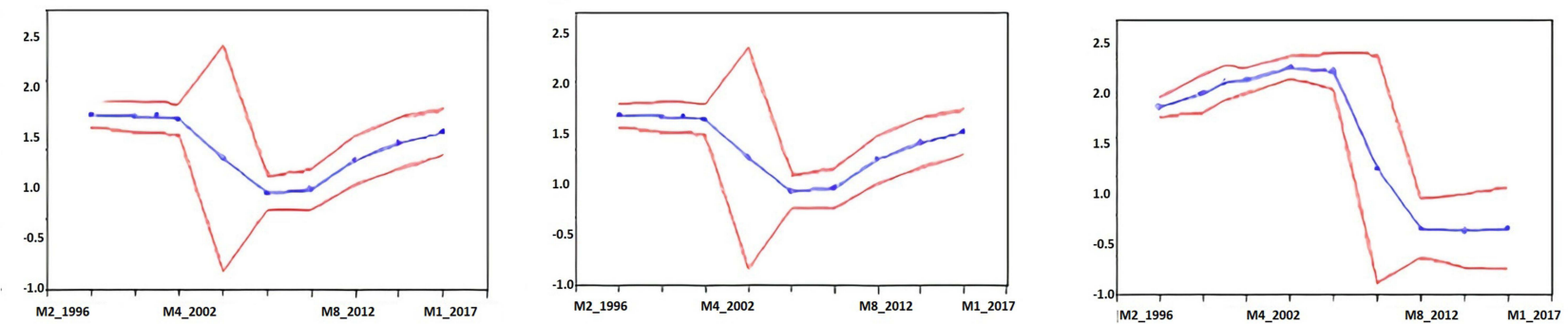

\section{HIV}
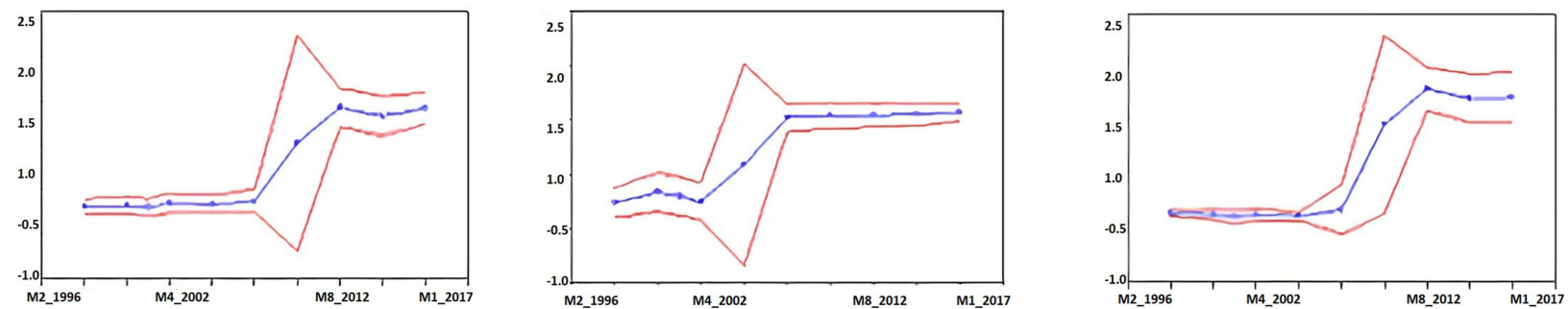

Pop
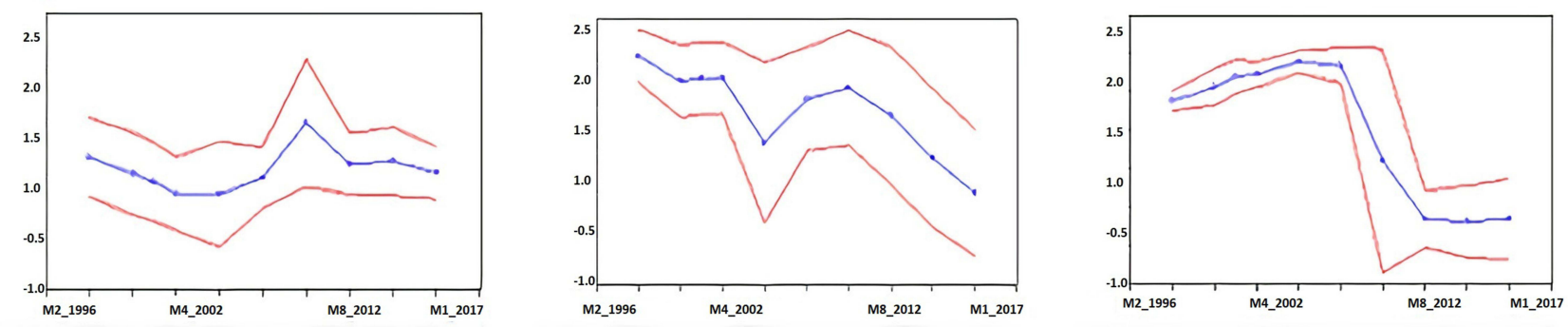

Figure I Rolling window QARDL estimation results - life expectancy.

Notes: In Figure I ECT(-I), GDP, HE, HIV and POP are estimated parameters using the rolling window method for Life Expectancy, and each window has 0 observations.

The $0.25,0.5,0.75$ are considered three different quantile levels. The $\mathrm{X}$-axis indicates the last date for the corresponding estimation window. 


\section{$\operatorname{ECT}(-1) \quad$ change in $p=0.25$,}

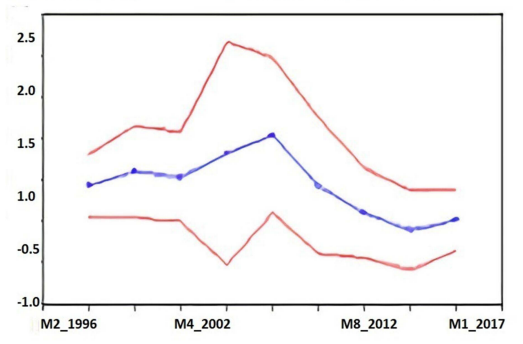

\section{GDP}

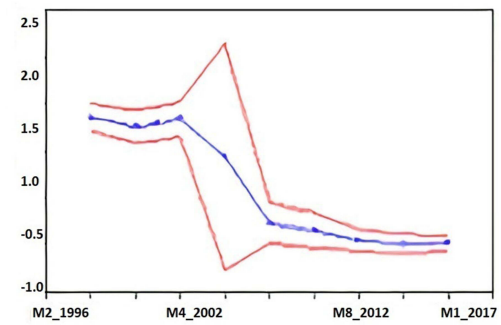

HE

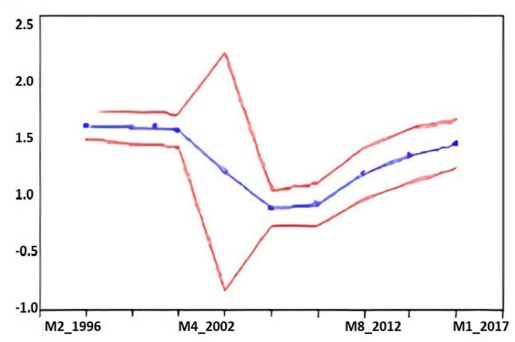

HIV

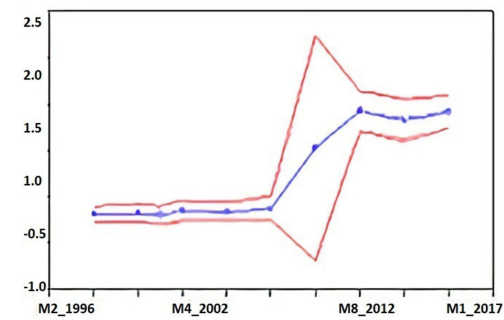

Pop

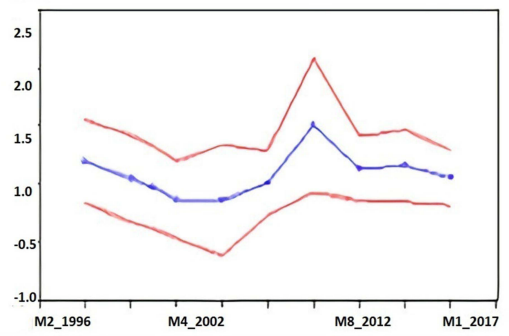

Change in $p=0.50$
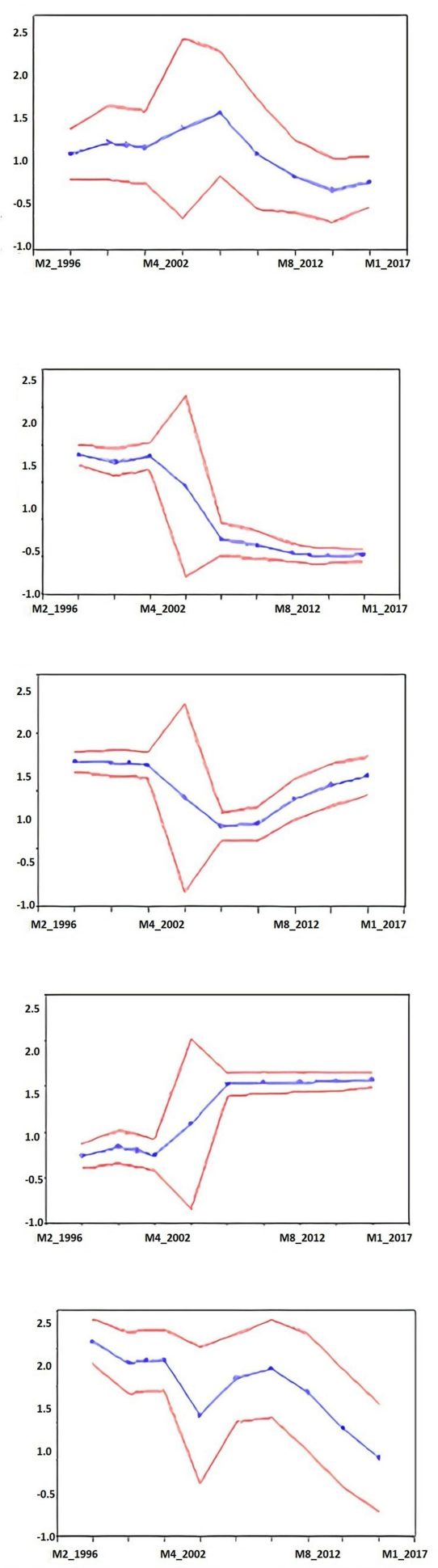

Change in $p=0.75$
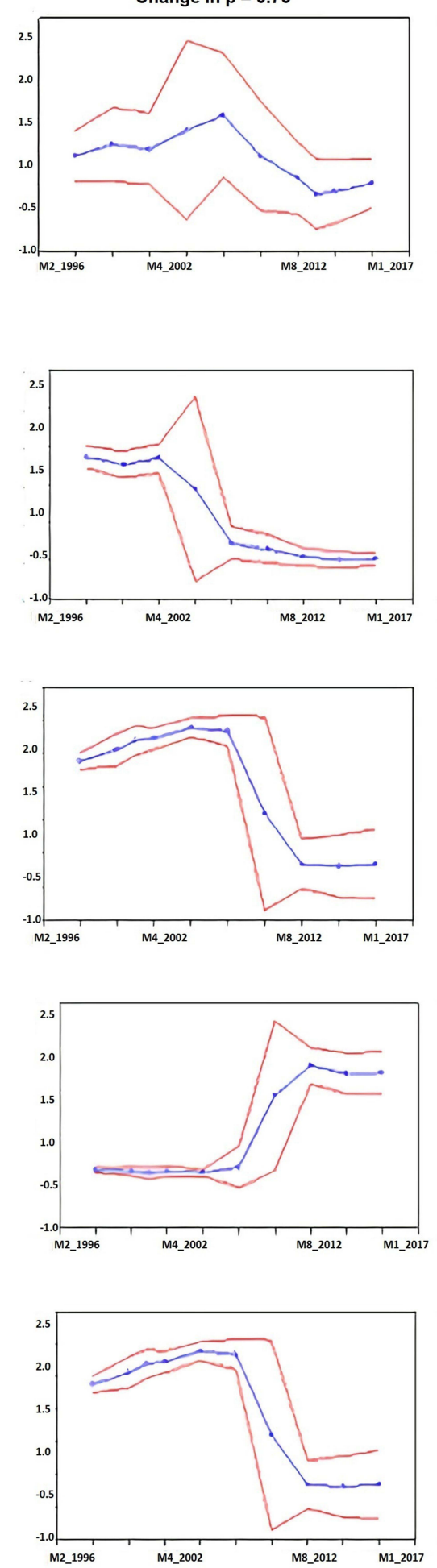

Figure 2 Rolling window QARDL estimation results - death rate.

Notes: In Figure 2 ECT(-I), GDP, HE, HIV and POP are estimated parameters using the rolling window method for Death rate, and each window has 0 observations. The $0.25,0.5,0.75$ are considered three different quantile levels. The $\mathrm{X}$-axis indicates the last date for the corresponding estimation window. 
$\operatorname{ECT}(-1) \quad$ Change in $p=0.25$,

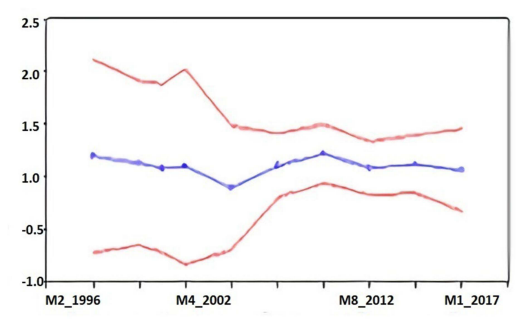

GDP

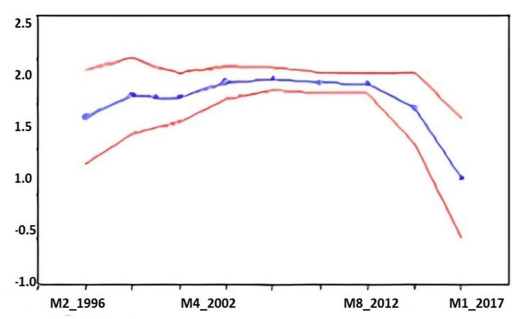

HE

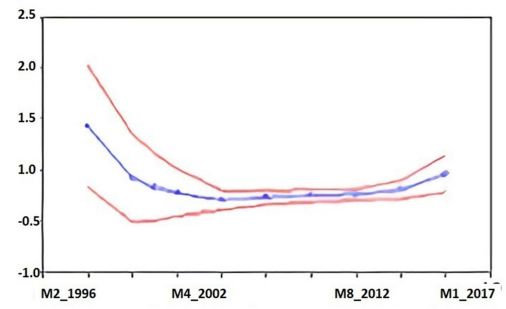

\section{HIV}

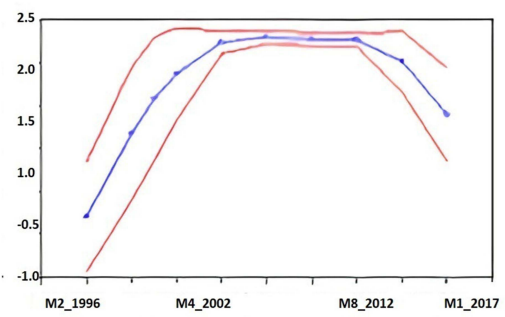

Pop

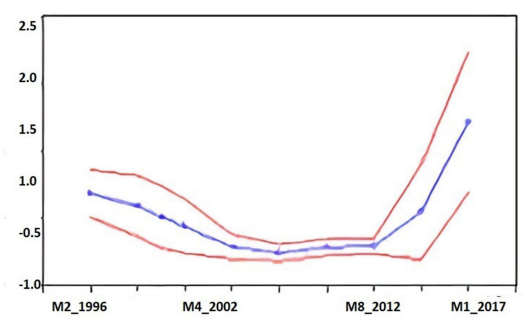

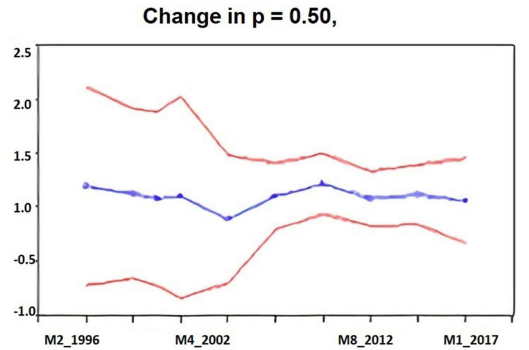
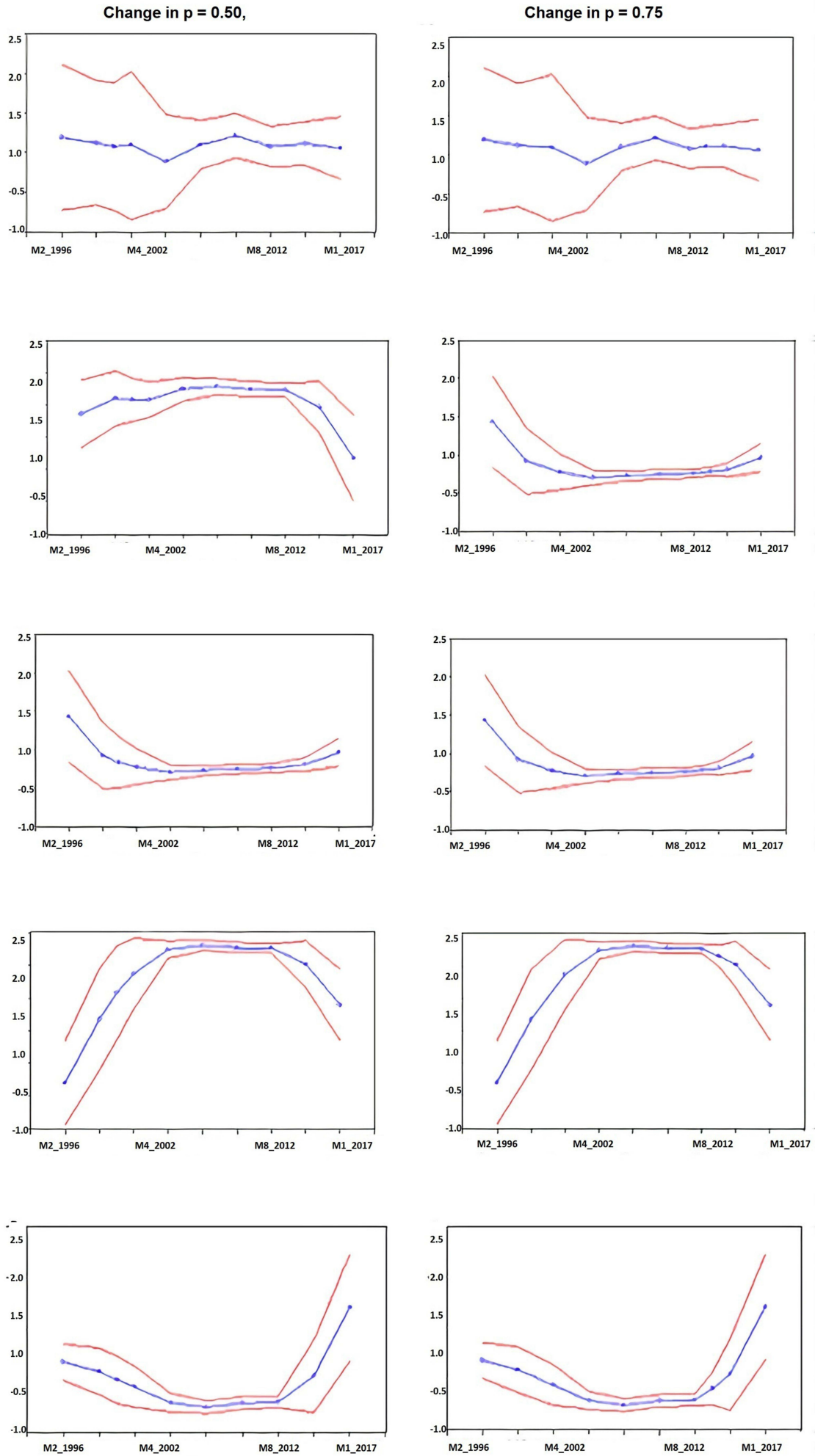

Figure 3 Rolling window QARDL estimation results - mortality.

Notes: In Figure 3 ECT(-I), GDP, HE, HIV and POP are estimated parameters using the rolling window method for mortality, and each window has 0 observations. The $0.25,0.5,0.75$ are considered three different quantile levels. The $X$-axis indicates the last date for the corresponding estimation window. 

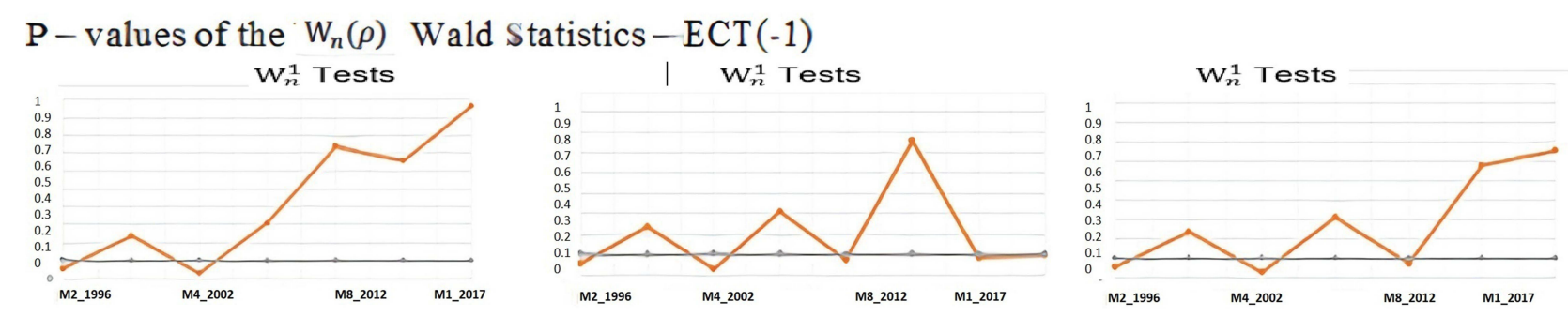

$\mathrm{P}$ - values of the $\mathrm{W}_{n}(\rho)$ Wald Statistics - GDP
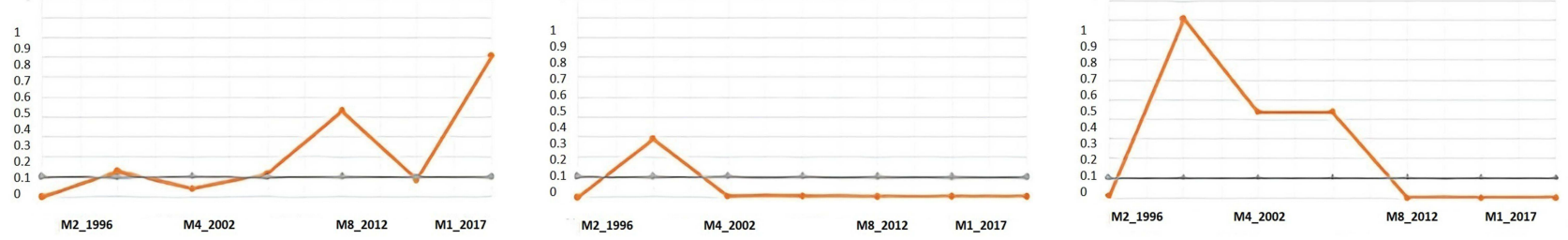

$\mathrm{P}$ - values of the $\mathrm{W}_{n}(\rho)$ Wald Statistics - HE
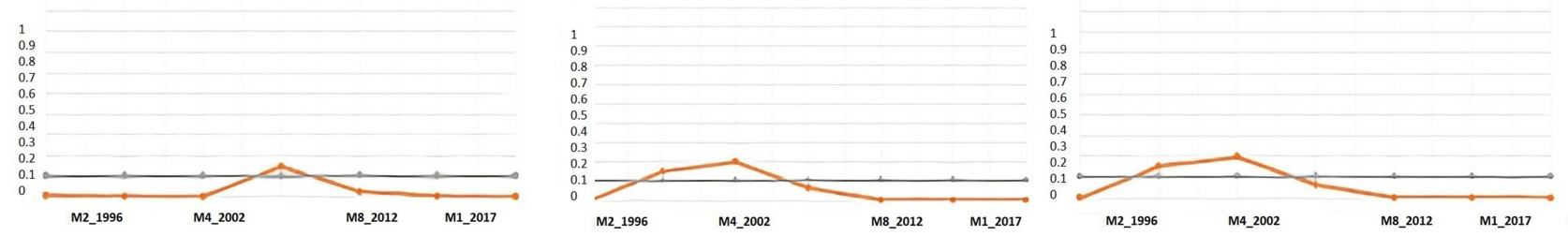

\section{$\mathrm{P}$ - values of the $\mathrm{W}_{n}(\rho)$ Wald Statistics - HIV}
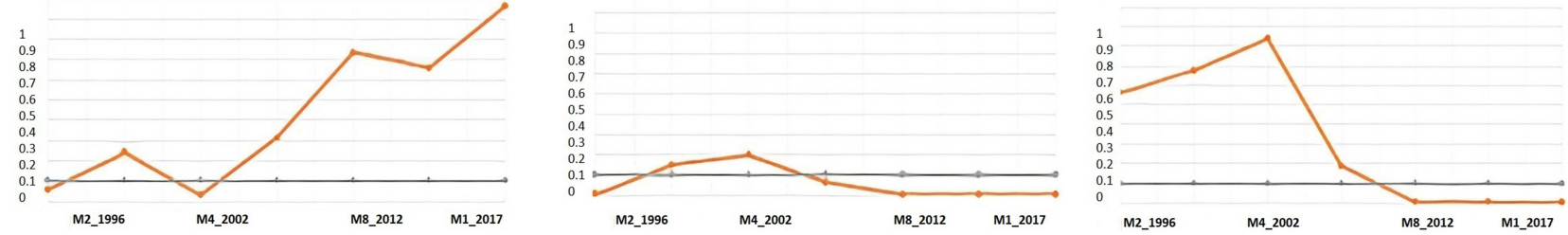

P - values of the $W_{n}(\rho)$ Wald Statistics - POP
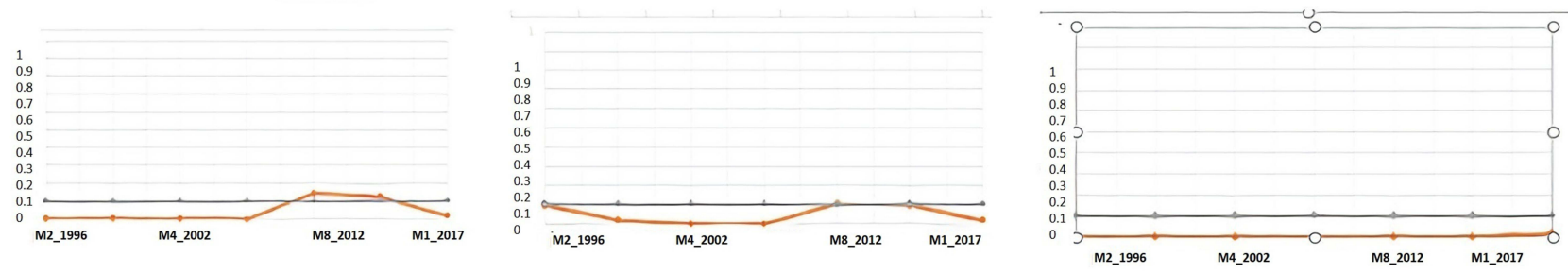

Figure 4 Rolling window results (life expectancy).

HIV in a number of people in the country. The population has a heterogeneous effect in short- and long estimates. In the short run, the population has a negative and significant impact on the life expectancy in all the quantiles, for instance in the 2 nd quantile, each percent increase in the population reduces the life expectancy by $0.04 \%$. This implies that a higher population leads to lower life expectancy. It puts pressure on the available public health and other necessary resources. Thus, it has an adverse implication. The run-long estimates show a positive and significant effect on most quantiles' life expectancy. In contrast, the first two quantiles show an insignificant effect. It implies that in the long run, the government has sufficient projects to ensure the basic health facilities 

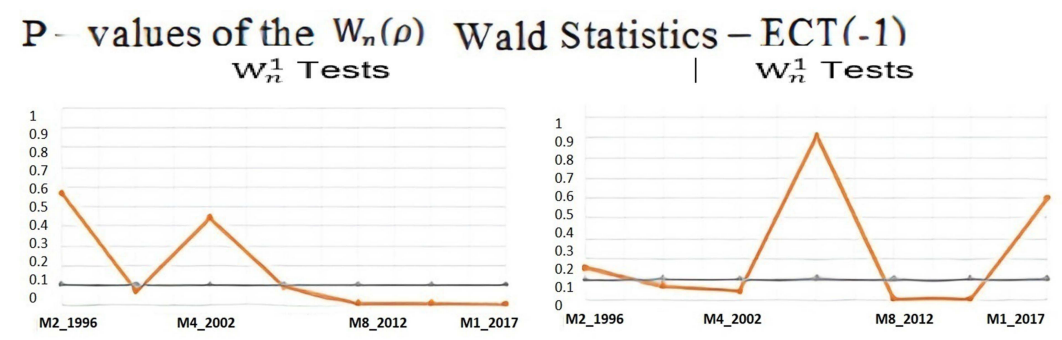

$\mathrm{P}$ - values of the $\mathrm{W}_{n}(\rho)$ Wald Statistics - GDP
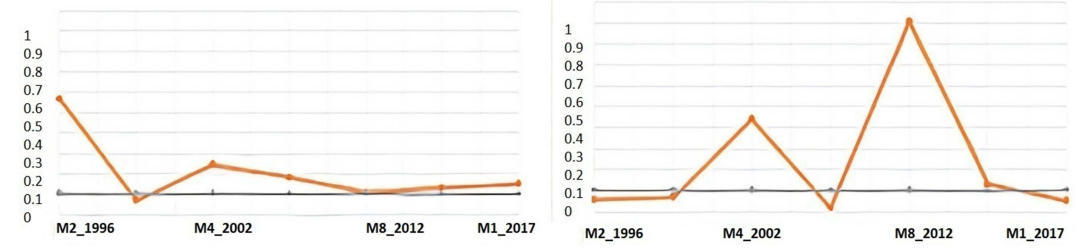

\section{$\mathrm{P}$ - values of the $\mathrm{W}_{n}(\rho)$ Wald Statistics - HE}
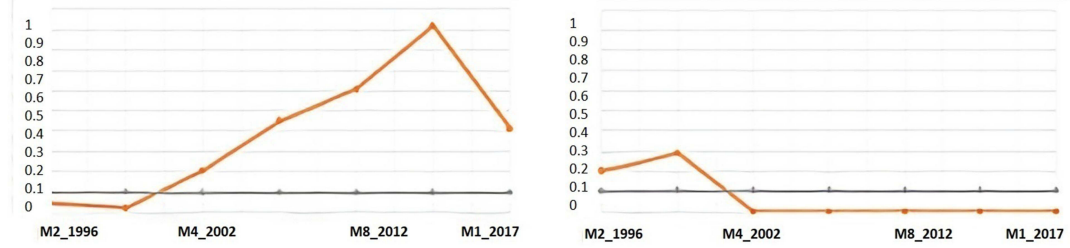

$\mathrm{P}$ - values of the $\mathrm{W}_{n}(\rho)$ Wald Statistics - HIV
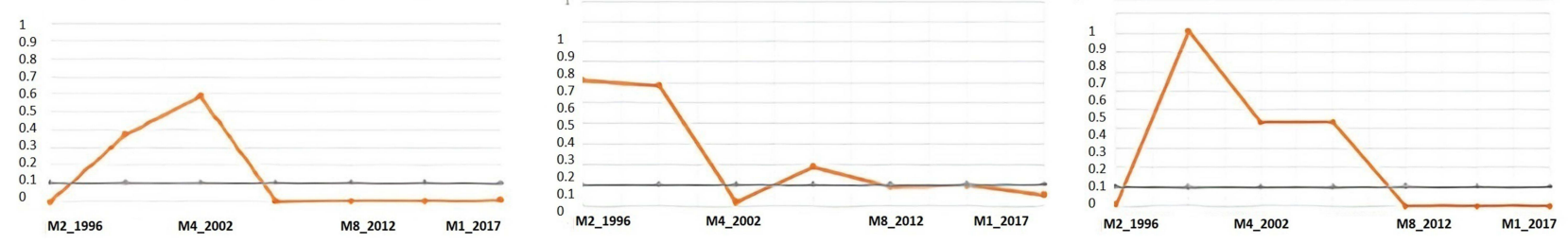

\section{$\mathrm{P}$ - values of the $\mathrm{W}_{n}(\rho)$ Wald Statistics - POP}
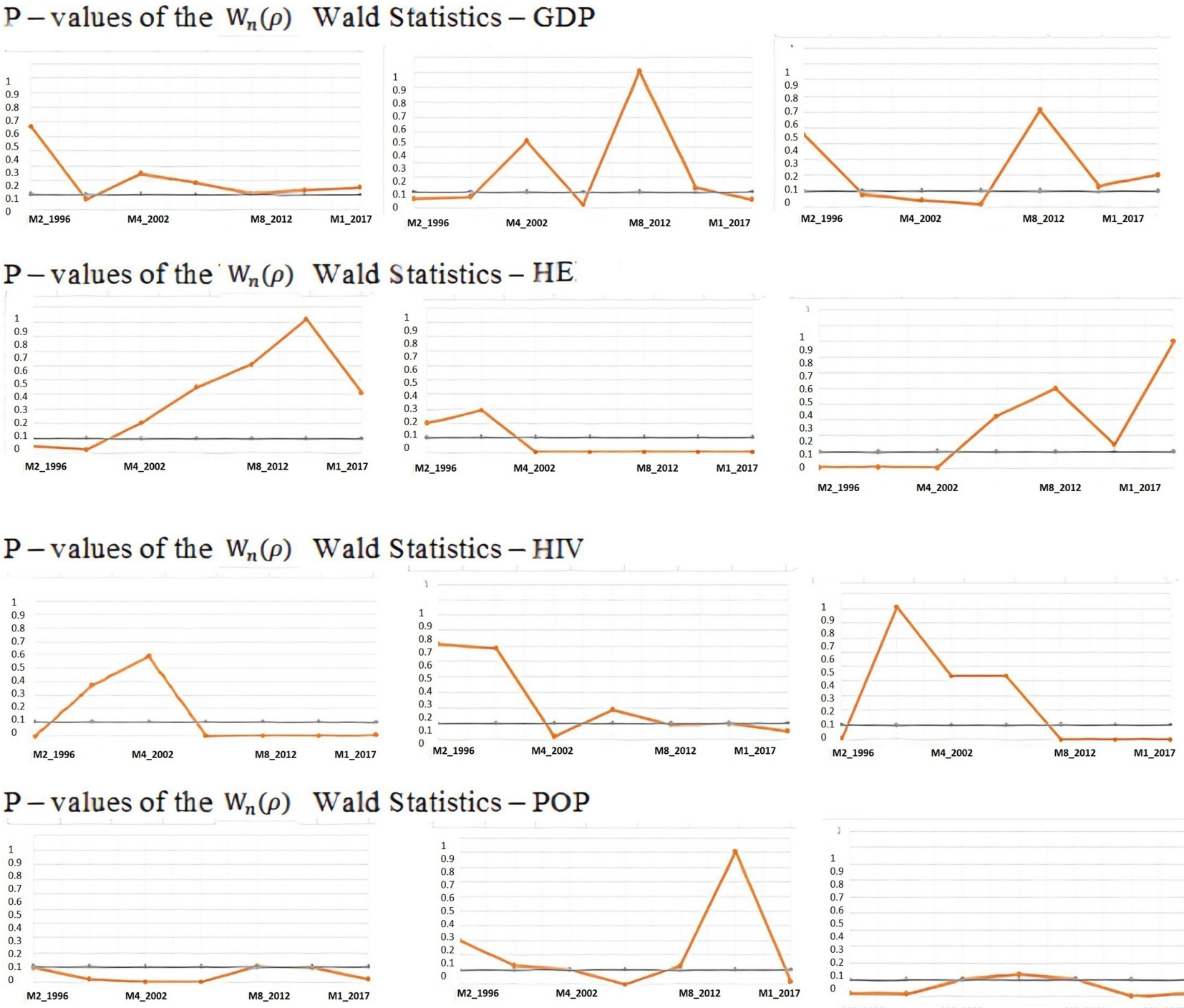

$W_{n}^{1}$ Tests

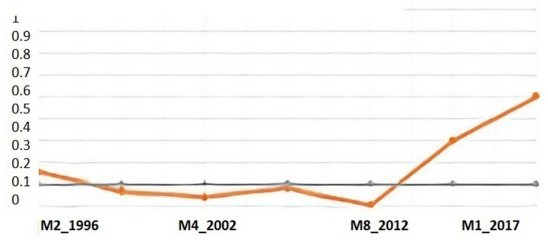

12017

Figure 5 Rolling window results (death rate).

including healthcare services; thus, the population, in the long run, depicts a positive effect on life expectancy.

In the second model, the death rate is the dependent variable. The error correction term for the model provides mixed results: some are positive, while some of these are positive, while some have a negative sign. Some of them were significant, whereas some remained insignificant. The 2nd, 3rd quantile, and 6th quantile values are insignificant, while the remaining quantiles have significant values. The significant coefficient of the error term implies a long-run association between the death rate and the included explanatory variables; however, most of the coefficient holds a positive sign. This means that short-run dynamics will achieve a long time span e to achieve longrun equilibrium. The per capita GDP holds a negative implication for death rate. However, in the 1 st to 4 th 

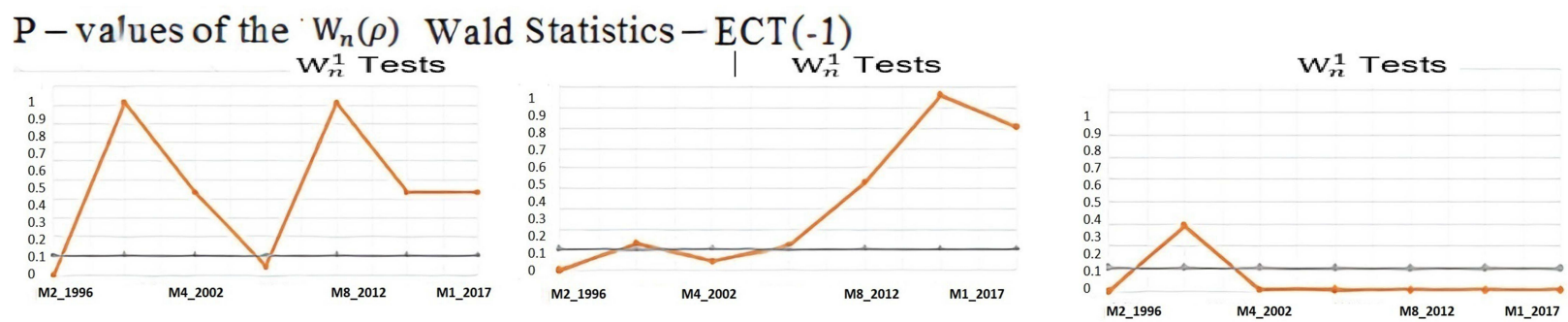

$\mathrm{P}$ - values of the $\mathrm{W}_{n}(\rho)$ Wald Statistics - GDP
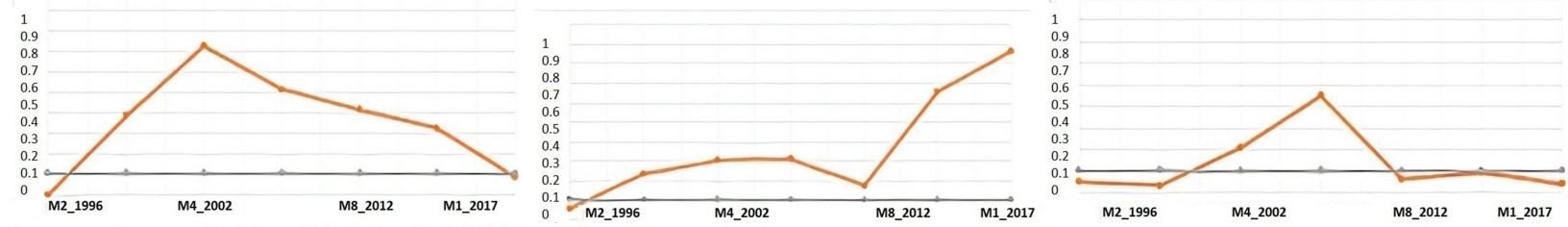

$\mathrm{P}$ - values of the $\mathrm{W}_{n}(\rho)$ Wald Statistics - HE
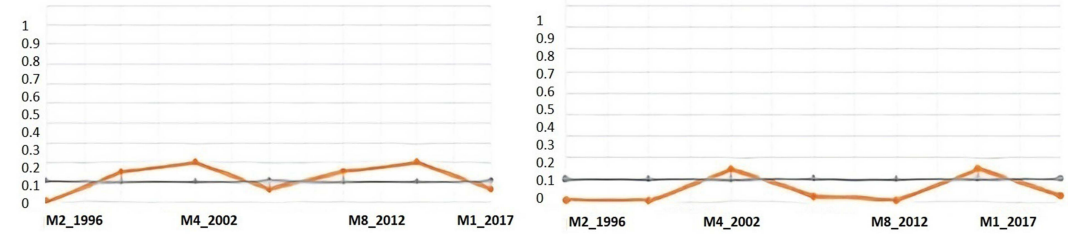

$\mathrm{P}$ - values of the $\mathrm{W}_{n}(\rho)$ Wald Statistics - HIV
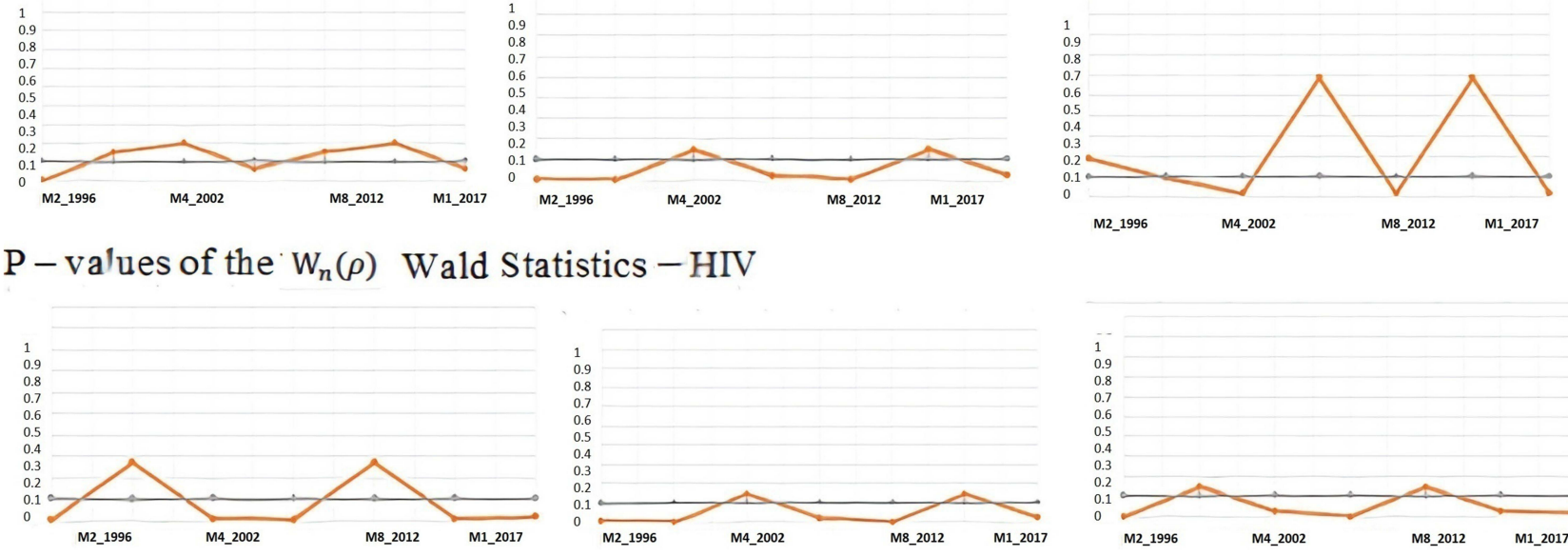

$\mathrm{P}$ - values of the $\mathrm{W}_{n}(\rho)$ Wald Statistics - POP
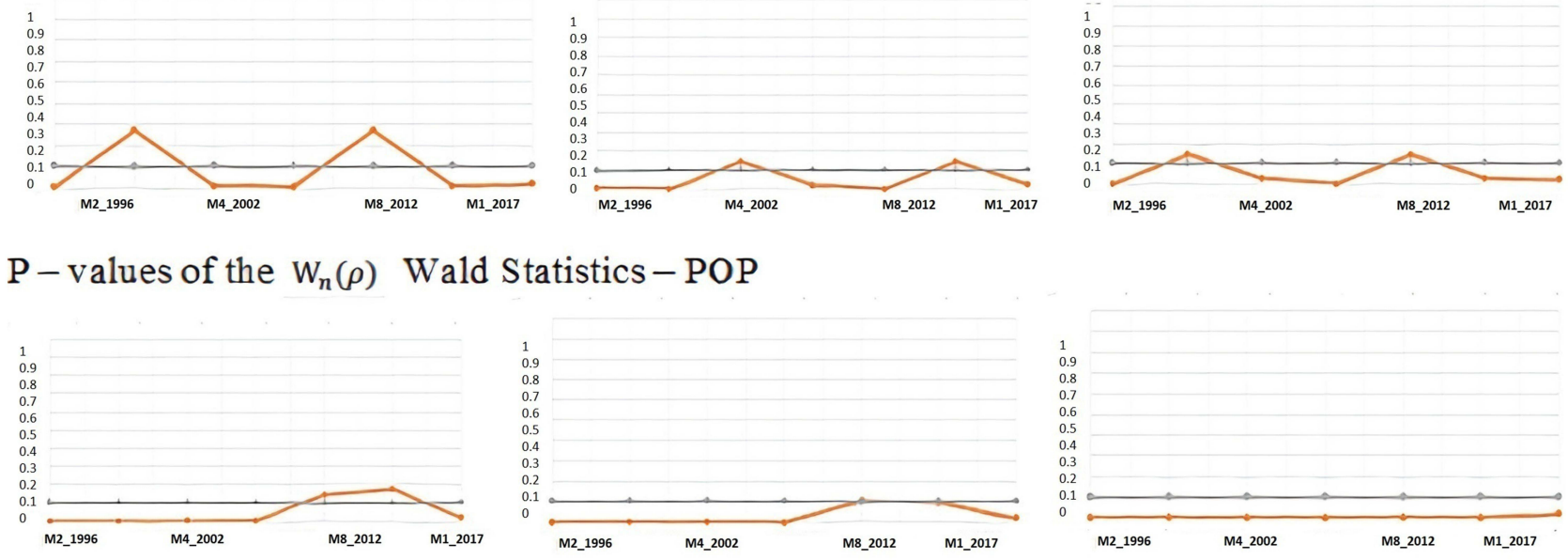

Figure 6 Rolling window results (mortality).

Notes: The figures show the estimated p-values of the Wald tests, where $\mathrm{W}_{n}^{1}$ tests $x_{*}=0.25$, $\mathrm{W}_{n}^{2}$ tests $x_{*}=0.50$ and $\mathrm{W}_{n}^{4}$ tests $x_{*}=0.75$. The horizontal axis represents the corresponding estimation.

quantile, the coefficient values are insignificant following the signs of all subsequent quantiles. An increase in GDP per capita reduces the death rate; for example, in the 4th quantile, each percent increase in GDP reduces the death rate in the country by 0.004 .

The long-run coefficients of GDP per capita are negative and significant except the 9th quantile, where the effect is insignificant. Such outcomes entail that GDP is partially determined by the death rate in the short run and strongly determined by the death rate in the long run, which means that the increase in income people has a more prosperity and well-being and no starvation, which considerably reduces the death rate. In addition, HIV is positively and significantly affecting the death rates both in the short and long run, except 
for a few quantiles, which hold insignificant signs. For example, 5th quantile and 6th quantiles in the short-run, while 7 th, 8th, and 9th quantile of long-run show insignificant. The magnitude values of the HIV coefficient are low, which indicates that though the reduction in HIV can be helpful to reduce the death rate due to the lower coefficient, it may contribute at a low level. The population has heterogeneous outcomes in the short and long run; as the population effect is insignificant in most quantiles in the short-run but has a positive and significant effect in the long run. This shows that population 'does not determine the death rate in the short run, while in the long run, a high population leads to more deaths in the country.

Table 6 entails the results for mortality. The error correction model outcomes show mixed outcomes. Some of them are positive, while others have a negative association with significant and insignificant coefficient as 1st quantile, 3rd quantile, 4th quantile is insignificant, while the rest of quantiles are significant. This significant coefficient implies longrun causality, but positive coefficients take a long time to achieve long-run equilibrium compared to negative signs. GDP per capita has negative and significant implications for mortality both in the short-run and long-run. However, the 1st and 2nd quantiles of the short run and 8th and 9th quantiles of the long run have an insignificant influence. Healthcare spending has a negative association with mortality; all quantiles show significant influence on mortality, but the 4 th and 5 th quantile of short-run and 8 th and 9 th quantiles of long-run reported an insignificant effect on mortality. This implies that healthcare spending reduces mortality; for example, in the 3 rd quantile in the long run, each percent increase in healthcare spending reduces mortality by $3.11 \%$.

These results imply that an increase in income reduces mortality in the country. People with sufficient income have access to the necessary health facilities and thus reduce mortality. HIV has a positive and significant effect on mortality both in the short-run and long-run. However, the 5 th and 6 th quantiles in the short-run and 6th, 7th, 8th, and 9th quantiles of short-run hold an insignificant coefficient. This shows mortality is more affected by HIV in the short-run rather than in the long run. The findings suggest that each percent increase in HIV increases mortality by 0.000438 in the 4 th quantile of the short run. The population has negatively influenced mortality both in the short and long runs. Almost all coefficients are significant in the short-run and the long-run parameters. Nonetheless, few of them are insignificant, for example, in the 4th and 5th quantiles of short-run and 8th quantile of the population are insignificant. The empirical finding suggests that the 7 th quantile of short-run shows that each percent increase in population increases the mortality.

Based on empirical findings, this study suggests some policy recommendations, since public health and expenditures can provide resources, improve the physical structure, and improve quality in the health sector. It is the responsibility of the public sector to facilitate and provide funds, ensure required healthcare staff, payment schemes, and incentives. Proper health caregiving facilities for patients would lead to health outcomes. Public health expenditures could provide technological improvement in the health sector and also result in better health outcomes. Based on the present study's findings, it is concluded that public health care expenditure enables the provision of better healthcare facilities, which leads to health outcomes and quality of health might have spillover effects on the growth and development of the country. Therefore, this study suggests that healthcare spending is an essential component of health outcomes, and the government should allocate sufficient funds for the healthcare sector. These findings contribute to the existing literature by providing short-run and long-run estimations for health spending and health outcomes. In addition, quantile ARDL approaches depict variations in health outcomes caused by the health spending in different quantiles and respective policy changes. The results of this study are in line with those of Osakede, ${ }^{30}$ Arthur, Oaikhenan, ${ }^{22}$ Ssozi, Amlani, ${ }^{23}$ and Nicholas et al. ${ }^{31}$

\section{Conclusions}

The deteriorating environmental quality resulting from rapid industrialization and increased emissions has adverse effects on human health and well-being. ${ }^{35,36,38}$ This, in turn, puts pressure on the governments to escalate their public health spending. ${ }^{37}$ Healthcare healthcare remained a major policy objective during different political regimes in Pakistan. The government introduced various healthcare programs that resulted in a substantial increase in health spending. The study findings reveal that health status indicators such as life expectancy, death rates, and mortality were mainly associated with public healthcare spending in Pakistan both in the short and long runs. We also found that public healthcare spending can increase life expectancy, and considerably reduce the country's mortality and death rate. This implies that public sector healthcare expenditures substantially improve health outcomes in Pakistan.

The outcomes of this study suggest the following policy recommendations: government should increase the health 
spending to optimize health outcomes especially in those regions where healthcare facilities are inadequate. Secondly, the government should upgrade the obsolete health infrastructure by employing the latest healthcare technologies to optimize the country's health further system for the development of a healthy society. Thirdly, longevity requires healthy life style and cleaner environment, therefore it is essential to adopt renewable energy technologies for pollution abatement. Hence, the concerned policymakers should draft policies to encourage biogas and solar photovoltaic energy technologies for pollution reduction and a greener economy. ${ }^{39}$ Such sustainable policy measures can lower the public health burden of the government and enhance the overall health outcomes of the country.

Lastly, against the backdrop of sluggish economic performance, the government has a limited cushion to escalate health care spending in Pakistan. The policy implications of this research entail that health care spending significantly promotes life expectancy and reduces the mortality rate in the country. Hence, the policymakers shall explore new avenues of investment in the health sector. Encouraging public-private partnerships and providing a conducive environment for private investors can lower the health spending burden of the government as well as boost the quality of life of the masses in the country.

Though this research also has some limitations: first, it explores the proposed association in the context of a single country - Pakistan. Second, we use time-series data till the year 2017 due to data availability constraints. Third, we only employ macroeconomic variables in our analysis. Future lines of research in this domain can be extended to multiple countries and regions, such as South Asian countries. In addition, the data period can be extended to account for different periods and political regimes in Pakistan. Furthermore, microeconomic variables such as household health spending can be incorporated by future research for an in-depth exploration of the underlying phenomenon.

\section{Funding}

This research is supported by the SPEV project 2021 at the Faculty of Informatics and Management, University of Hradec Kralove, Czech Republic.

\section{Disclosure}

The authors report no conflicts of interest in this work.

\section{References}

1. Romer D. Advanced Macroeconomics. McMGrawMHill Companies. Inc Singapore.; 1996.

2. Filmer D. Child Mortality and Public Spending on Health: How Much Does Money Matter? The World Bank; 1999.

3. Ali A, Ahmad K The impact of socio-economic factors on life expectancy for sultanate of Oman: an empirical analysis; 2014. Available form: https://mpra.ub.uni-muenchen.de/70871/1/MPRA paper_70871.pdf. Accessed September 10, 2021.

4. Somi MF, Butler JR, Vahid F, Njau JD, Abdulla S. Household responses to health risks and shocks: a study from rural Tanzania raises some methodological issues. J Int Dev. 2009;21(2):200-211. doi:10.1002/jid. 1555

5. Shah MH, Wang N, Ullah I, Akbar A, Khan K, Bah K. Does environment quality and public spending on environment promote life expectancy in China? Evidence from a nonlinear autoregressive distributed lag approach. Int J Health Plann Manage. 2021;36 (2):545-560. doi:10.1002/hpm.3100

6. Ostro BD, Rothschild S. Air pollution and acute respiratory morbidity: an observational study of multiple pollutants. Environ Res. 1989;50(2):238-247. doi:10.1016/S0013-9351(89)80004-0

7. Schwartz J, Dockery DW. Increased mortality in Philadelphia associated with daily air pollution concentrations. Am Rev Respir Dis. 1992;145(3):600-604. doi:10.1164/ajrccm/145.3.600

8. Hatfield LA, Favreault MM, McGuire TG, Chernew ME. Modeling health care spending growth of older adults. Health Serv Res. 2018;53(1):138-155. doi:10.1111/1475-6773.12640

9. Apergis N, Gupta R, Lau CKM, Mukherjee Z. US state-level carbon dioxide emissions: does it affect health care expenditure? Renew Sustain Energy Rev. 2018;91:521-530. doi:10.1016/j. rser.2018.03.035

10. Hansen AC, Selte HK. Air pollution and sick-leaves. Environ Resour Econ. 2000;16(1):31-50. doi:10.1023/A:1008318004154

11. Jerrett M, Eyles J, Dufournaud C, Birch S. Environmental influences on healthcare expenditures: an exploratory analysis from Ontario, Canada. J Epidemiol Community Health. 2003;57(5):334-338. doi:10.1136/jech.57.5.334

12. Chaabouni S, Saidi K. The dynamic links between carbon dioxide (CO2) emissions, health spending and GDP growth: a case study for 51 countries. Environ Res. 2017;158:137-144. doi:10.1016/j. envres.2017.05.041

13. Brunekreef B, Holgate ST. Air pollution and health. Lancet. 2002;360 (9341):1233-1242. doi:10.1016/S0140-6736(02)11274-8

14. Mead RW, Brajer V. Protecting China's children: valuing the health impacts of reduced air pollution in Chinese cities. Environ Dev Econ. 2005;10(6):745-768. doi:10.1017/S13557 70X05002512

15. Narayan PK, Narayan S. Carbon dioxide emissions and economic growth: panel data evidence from developing countries. Energy Policy. 2010;38(1):661-666. doi:10.1016/j.enpol.2009.09.005

16. Janke K, Propper C, Henderson J. Do current levels of air pollution kill? The impact of air pollution on population mortality in England. Health Econ. 2009;18(9):1031-1055. doi: $10.1002 /$ hec. 1475

17. Remoundou K, Koundouri P. Environmental effects on public health: an economic perspective. Int J Environ Res Public Health. 2009;6 (8):2160-2178. doi:10.3390/ijerph6082160

18. Beatty TK, Shimshack JP. Air pollution and children's respiratory health: a cohort analysis. J Environ Econ Manage. 2014;67(1):39-57. doi:10.1016/j.jeem.2013.10.002

19. Akinkugbe O, Mohanoe M. Public health expenditure as a determinant of health status in Lesotho. Soc Work Public Health. 2009;24(1-2):131-147. doi:10.1080/19371910802569716 
20. Novignon J, Olakojo SA, Nonvignon J. The effects of public and private health care expenditure on health status in sub-Saharan Africa: new evidence from panel data analysis. Health Econ Rev. 2012;2(1):22. doi:10.1186/2191-1991-2-22

21. Ventelou B, Abu-Zaineh M. Medicine and democracy: the importance of institutional quality in the relationship between health expenditure and health outcomes in the MENA region. Health Policy (New York). 2016;120(8):928-935. doi:10.1016/j.healthpol.20 16.06.005

22. Arthur E, Oaikhenan HE. The effects of health expenditure on health outcomes in Sub-Saharan Africa (SSA). Afr Dev Rev. 2017;29 (3):524-536. doi:10.1111/1467-8268.12287

23. Ssozi J, Amlani S. The effectiveness of health expenditure on the proximate and ultimate goals of healthcare in Sub-Saharan Africa. World Dev. 2015;76:165-179. doi:10.1016/j.worlddev.2015.07.010

24. Behera DK, Dash U. Is health expenditure effective for achieving healthcare goals? Empirical evidence from South-East Asia Region. Asia Pac J Reg Sci. 2020;4(2):593-618. doi:10.1007/s41685-02000158-4

25. Dollar D, Burnside C. Aid, the Incentive Regime, and Poverty Reduction. The World Bank; 1999.

26. Bokhari FA, Gai Y, Gottret P. Government health expenditures and health outcomes. Health Econ. 2007;16(3):257-273. doi:10.1002/ hec. 1157

27. Anyanwu JC, Erhijakpor AE. Health expenditures and health outcomes in Africa. Afr Dev Rev. 2009;21(2):400-433. doi:10.1111/ j.1467-8268.2009.00215.x

28. Akram M, Khan FJ. Health Care Services and Government Spending in Pakistan. East Asian Bureau of Economic Research; 2007.

29. Chaudhry MO, Zahir M, Farooq F, Arif R. Contribution of Health Outcomes to Economic Growth in Pakistan. Pak J Soc Sci. 2013;33(2):281-295.

30. Osakede UA. Public health spending and health outcome in Nigeria: the role of governance. Int $J$ Dev Issues. 2020;20:95-112. doi:10.1108/IJDI-10-2019-0169
31. Nicholas A, Edward N-A, Bernardin S. The effect of health expenditure on selected maternal and child health outcomes in Sub-Saharan Africa. Int J Soc Econ. 2016;43(12):1386-1399. doi:10.1108/IJSE08-2015-0199

32. Zhu H, Peng C, You W. Quantile behaviour of cointegration between silver and gold prices. Fin Res Lett. 2016;19:119-125. doi:10.1016/j. frl.2016.07.002

33. Pesaran MH, Shin Y. An autoregressive distributed-lag modelling approach to cointegration analysis. Econometric Soc Monogr. 1998;31:371-413.

34. Mensi W, Shahzad SJH, Hammoudeh S, Hkiri B, Al Yahyaee KH. Long-run relationships between US financial credit markets and risk factors: evidence from the quantile ARDL approach. Fin Res Lett. 2019;29:101-110. doi:10.1016/j.frl.2019.03.007

35. Shahbaz M, Lahiani A, Abosedra S, Hammoudeh S. The role of globalization in energy consumption: a quantile cointegrating regression approach. Energy Econ. 2018;71:161-170. doi:10.1016/j. eneco.2018.02.009

36. Bell BS, Kozlowski SW. Adaptive guidance: enhancing self-regulation, knowledge, and performance in technology-based training. Pers Psychol. 2002;55(2):267-306. doi:10.1111/j.1744-6570.2002.tb00111.x

37. Johansen S. Likelihood-Based Inference in Cointegrated Vector Autoregressive Models. Oxford University Press on Demand; 1995.

38. Akbar M, Hussain A, Akbar A, Ullah I. The dynamic association between healthcare spending, $\mathrm{CO} 2$ emissions, and human development index in OECD countries: evidence from panel VAR model. Environ Dev Sustain. 2021;23(7):10470-10489. doi:10.1007/s10668020-01066-5

39. Akbar A, Alam Rehman IU, Zeeshan M, Afridi FEA. Unraveling the dynamic nexus between trade liberalization, energy consumption, $\mathrm{CO} 2$ emissions, and health expenditure in Southeast Asian Countries. Risk Manag Healthc Policy. 2020;13:1915. doi:10.2147 RMHP.S272801
Risk Management and Healthcare Policy

\section{Publish your work in this journal}

Risk Management and Healthcare Policy is an international, peerreviewed, open access journal focusing on all aspects of public health, policy, and preventative measures to promote good health and improve morbidity and mortality in the population. The journal welcomes submitted papers covering original research, basic science, clinical \& epidemiological studies, reviews and evaluations, guidelines, expert opinion and commentary, case reports and extended reports. The manuscript management system is completely online and includes a very quick and fair peer-review system, which is all easy to use. Visit http://www.dovepress.com/testimonials.php to read real quotes from published authors. 